\title{
Molecular Dynamics Test of the Stress-Thermal Rule in Polyethylene and Polystyrene Entangled Melts
}

\author{
David Nieto Simavilla,* Aristotelis P. Sgouros, Georgios G. Vogiatzis, Christos Tzoumanekas, \\ Vasilis Georgilas, Wilco M. H. Verbeeten, and Doros N. Theodorou
}

Cite This: Macromolecules 2020, 53, 789-802

Read Online

ACCESS

Llll Metrics \& More

Article Recommendations

Supporting Information

ABSTRACT: Anisotropic thermal transport induced by deformation and the linear relation between the thermal conductivity and stress tensors, also known as the stress-thermal rule (STR), are tested via molecular dynamics simulations in well-entangled linear polyethylene (PE) and polystyrene (PS) melts subjected to extensional flow. We propose a method to determine the stress in deformed molecular melts, a key component missing in prior simulation studies on thermal transport in polymers that prevented verification of the STR. We compare our results with available data from previous experimental and simulation studies. Thermal conductivity (TC) is found to increase (decrease) in the direction

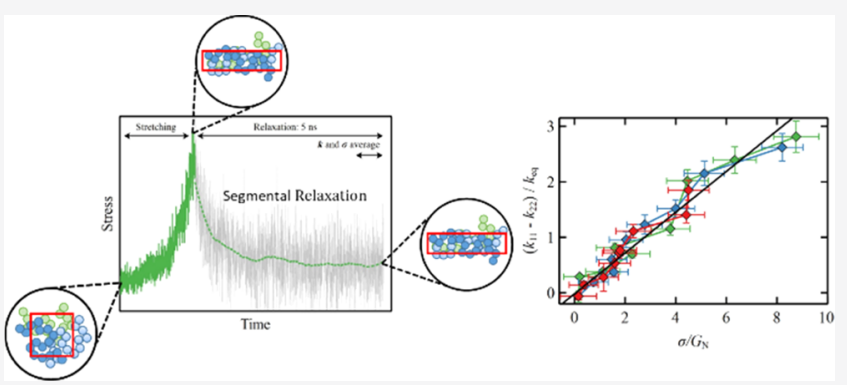
parallel (perpendicular) to the imposed stretch. We find that the STR is valid for both PE and PS over a wide range of deformation rates and stress levels. In direct agreement with experimental evidence and the STR, we observe that for a given strain, the anisotropy in TC increases with the strain rate. Surprisingly, our results for PE question the universal behavior with respect to polymer chemistry suggested by experiments by showing a significantly higher proportionality constant (the stress-thermal coefficient) between stress and anisotropy in TC. We argue that this discrepancy can be explained by the high degree of entanglement interactions in PE affecting the transport of energy at the molecular level. Our conjecture is tested by studying an entangled linear PS melt, a polymer with a much lower entanglement plateau, for which thermal transport experimental results are available. For PS, the normalized stress-thermal coefficient is found to be commensurate with the experimental value. Finally, we test the fundamental molecular hypothesis of preferential energy transport along the backbone of polymer chains used to formulate the STR, which was prompted by early experimental evidence showing an increase in TC with chain length. We are able to establish that the increase in TC with chain length in PE melts fades as the system becomes entangled (i.e., TC remains constant beyond the critical entanglement chain length that marks the transition to entanglement-dominated rheological behavior). Our findings are of key importance in developing robust molecular-to-continuum methodologies for the study of nonisothermal macroscopic flows that are extremely relevant to polymer manufacturing processes.

\section{INTRODUCTION}

Computational fluid dynamics (CFD) simulations are becoming widespread in the study of manufacturing processes involving polymeric material flows. This tool can help connect the final properties of components to process parameters and represents a more cost-effective approach to process and product design than traditional experimentation. ${ }^{1}$ However, proper simulations of polymeric flows require careful analysis of the constitutive equations governing rheological and thermophysical properties. In this regard, significant progress has been made by implementing quantitative tube-based macroscopic network models (e.g., eXtended Pom-Pom, ${ }^{2}$ Rolie-Poly, ${ }^{3}$ and Molecular Stress Function ${ }^{4}$ among others) able to describe the rheology of polymeric melts in CFD packages. ${ }^{5,6}$ Surprisingly, the inherent nonisothermal character of polymer processing flows has been rarely discussed., ${ }^{6,7}$ Furthermore, the strong coupling between thermophysical properties, such as thermal conductivity (TC), and rheological behavior is often neglected. ${ }^{1,5,8-10}$ Experimental studies have shown that TC becomes anisotropic in polymer melts and networks subjected to deformation. ${ }^{11-13}$ For this phenomenon to be studied, Fourier's law is generalized for anisotropic materials. The thermal conductivity, typically considered an isotropic property function of temperature only (i.e., represented by a scalar function $k=k(T)$ ), becomes a tensorial property $\mathbf{k}=\mathbf{k}(T, \boldsymbol{\tau})$ that depends on temperature and stress due to the molecular reorientation of polymer chain segments. ${ }^{14}$ This could be a cause for concern, since the

Received: October 2, 2019

Revised: December 20, 2019

Published: February 3, 2020 
dependence of the TC tensor $\mathbf{k}$ on the stress tensor $\boldsymbol{\tau}$ further couples the energy and momentum balances. Simultaneous numerical determination of the velocity and temperature fields is a challenging problem in the simulation of polymeric flows due to the complexity of the constitutive equations needed to describe the rheology of these systems. Fortunately, using simple molecular arguments, van den Brule proposed a linear relationship between thermal conductivity and stress, known as the stress-thermal rule (STR) ${ }^{14}$

$$
\mathbf{k}-k_{\mathrm{eq}} \boldsymbol{\delta}=k_{\mathrm{eq}} C_{\mathrm{t}} \boldsymbol{\tau}
$$

where the proportionality constant $C_{t}$ is the stress-thermal coefficient and $k_{\text {eq }}=1 / 3 \operatorname{tr}\left(\mathbf{k}_{\mathrm{eq}}\right)$ is equilibrium TC of the unstretched system. Note that in eq 1 , we have taken $\tau$ to be the extra stress (i.e., $\boldsymbol{\tau}=\boldsymbol{\Pi}-1 / 3 \operatorname{tr}(\boldsymbol{\Pi}) \boldsymbol{\delta}$ with $\boldsymbol{\Pi}$ being the total stress tensor and $\boldsymbol{\delta}$ being the unit tensor). The proportionality expressed by eq 1 indicates that the anisotropy in thermal conductivity is related to the deviation from the equilibrium structure that gives rise to the stress. The STR is based on a simple molecular argument: thermal energy is preferentially transmitted along the backbone of a polymer chain. This hypothesis can be based on the disparity between the stiffness of $\mathrm{C}-\mathrm{C}$ bonds $(>250 \mathrm{GPa})$ and nonbonded interactions $(\sim 100 \mathrm{MPa}){ }^{15}$ Furthermore, the hypothesis is supported by both experimental and simulation studies of the molecular weight (or chain length) dependence of thermal conductivity. ${ }^{16-19}$ However, the limits to the relationship between TC and the molecular weight in a polymeric melt beyond the onset of entanglements have not been fully addressed.

Significant experimental ${ }^{11-13}$ and simulation ${ }^{20-26}$ efforts have been dedicated to studying the TC in polymers and the possibility of tuning it using deformation of the molecular structure. Of particular relevance to our work are the simulation studies on the TC of uniaxially stretched polystyrene by Algaer et al. $^{24}$ and Terao et al. ${ }^{25}$ and of polyethylene by Liu et al. ${ }^{26}$ for the similarity of their systems to the ones in our study. However, the above-mentioned studies of the anisotropy in TC induced by deformation failed to characterize the stress in deformed samples, making it impossible to test the STR. Furthermore, the study by Liu et $\mathrm{al}^{26}$ found that anisotropy in TC decreases with increasing strain rate in systems stretched below the glass-transition temperature. Their results suggest a negative stress-thermal coefficient in accordance with an experimental study on glassy PS samples stressed at room temperature by Gupta et al. ${ }^{27}$ An analysis of this set of results was offered by Schieber et al. ${ }^{28}$ using the framework of the minimum thermal conductivity (MTC) model. ${ }^{29}$ Following this same framework, the proportionality between TC and the sound velocities or the material stiffness has been shown to hold for rather complex polymeric systems including water-soluble polymers ${ }^{30}$ and blends. ${ }^{30,31}$ A recent study by Venerus et al. ${ }^{32}$ discusses the need of a generalization of the MTC model connecting the variations in the speed of sound of stretched elastomers to the observed anisotropy in TC. Note that above the glasstransition temperature, the experimental results for the same material suggest positive stress-thermal coefficients in polymer melts subjected to uniaxial extension ${ }^{27}$ (i.e., for a given deformation, a higher strain rate typically results in higher stress, and therefore according to the STR, one will expect higher anisotropy in the TC tensor). In this study, we focus on amorphous or disordered systems representing polymeric melts above the glass-transition temperature (i.e., systems that do not undergo crystallization in the presence or absence of strain). It has been shown elsewhere that both TC and heat capacity of polymeric materials are strongly affected by crystallization. ${ }^{33}$ Furthermore, to avoid effects such as crazing or void formation in the strained melts, we limit our study to moderate strain rates and strains as indicated by the straininduced crystallization study by Sliozberg et al. ${ }^{34}$ In addition, we acknowledge that the window of strain rates accessible by nonequilibrium molecular dynamics (NEMD) is significantly higher than the experimentally accessible range, making direct comparison with experiments rather challenging.

Recent experimental studies by Venerus and co-workers used two novel techniques (forced Rayleigh scattering (FRS) and infrared thermography (IRT)) to characterize the anisotropy in thermal conductivity ${ }^{27,28,35-37}$ and the stress in polymers subjected to deformation. Their work constitutes, to date, the most comprehensive experimental validation of the STR for uniaxially stretched melts ${ }^{27,28}$ and cross-linked elastomers. $^{35-37}$ Two additional key findings from these experimental studies are remarkably useful in modeling anisotropy in thermal conductivity induced by deformation.

(1) Universality of the normalized stress-thermal coefficient, $C_{\mathrm{t}} G_{\mathrm{N}} \simeq 0.04$ (i.e., the proportionality constant, once normalized by the entanglement plateau $G_{N}$ characteristic of the material, becomes independent of polymer chemical constitution). ${ }^{27,28,35-38}$

(2) Wide range of application in contrast to the stress-optic rule (SOR), it has been shown that the stress-thermal rule holds even after the finite extensibility of the polymer chain segments is reached. ${ }^{36}$ This result puts into question the molecular argument that anisotropy is a result of the orientation of chain segments along the strain direction only (i.e., further loading/stressing a "fully" oriented/stretched sample results in additional anisotropy following the STR).

While the STR can be explained using simple molecular arguments, ${ }^{14}$ the findings described in (1) and (2) are not well understood. The challenge in developing a consistent model for heat transport in polymeric materials is that, due to their complex and highly disordered structure, phonon-transport theories for neither solids nor liquids can be applied directly. ${ }^{39,40}$ To tackle this challenge, we propose using molecular simulation to first reproduce and extend the available experimental results and, second, discern the mechanisms and structural features governing anisotropic thermal transport induced by deformation in polymers.

In this study, we use molecular simulation techniques to determine anisotropy in thermal conductivity and stress induced by deformation of model melts with the goal of testing the STR. Universality of the STR is tested by comparing results for model polyethylene and polystyrene melts over a wide range of strains, strain rates, and stress levels. An advantage of studying these two polymeric materials is that they have been the target of numerous simulation studies due to their importance to the plastics industry. As a result, welldefined force fields able to describe the equilibrium structure and a plethora of other properties ${ }^{41,42}$ have been developed and thoroughly tested. The melts are subjected to uniaxial extension through nonequilibrium molecular dynamics (NEMD) simulations. Several configurations are extracted during the stretching of samples to study both stress and 


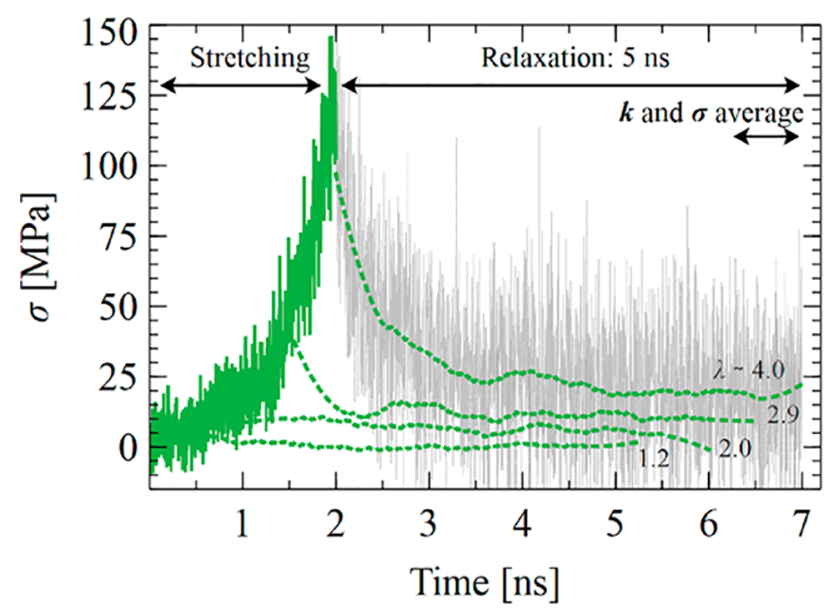

A

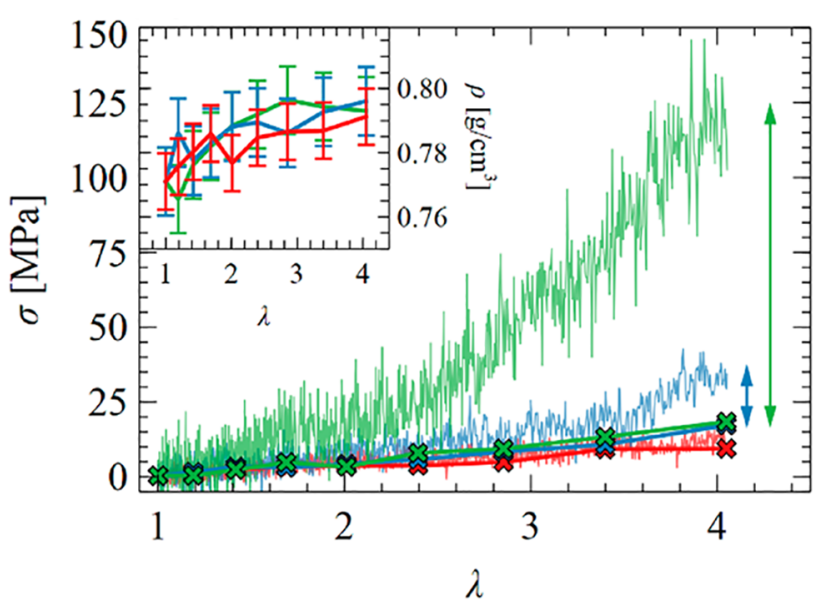

B

Figure 1. Stress determination approach for PE $8 \times 1000$. (A) Stress as a function of time during NEMD stretching at the highest Wi $=3500$ (green solid line). Stress moving average of the subsequent stress relaxation for a selection of configurations with stretch ratios $\lambda \simeq 1.2,2.0,2.9$, and 4.0 (green dashed lines). Example of raw stress data without averaging for the highest strain (gray solid line). (B) Comparison of the stress computed during NEMD stretching (translucent solid lines without symbols) and the relaxed stress averaged over the last $0.5 \mathrm{~ns}$ of $5 \mathrm{~ns}$ poststretching isochoric relaxation (cross symbols) at $\mathrm{Wi}=50$ (red), $\mathrm{Wi}=500$ (blue), and $\mathrm{Wi}=3500$ (green). The inset shows the density (averaged over the same window as the relaxed stress) as a function of the stretch ratio. Error bars are based on the standard deviation. All simulations were performed at $450 \mathrm{~K}$.

anisotropy in TC induced by deformation. In addition, we investigate the dependence of thermal conductivity on the molecular weight of polyethylene, which indirectly tests the hypothesis of preferential energy transport along the chain backbones. Our findings highlight the importance of entanglements as a key structural feature to explain the mechanisms governing thermal transport and leading to anisotropic thermal conductivity in polymers.

Simulation Methods. We have studied TC and stress of two model monodisperse linear melts of significantly different chemical constitutions (polyethylene and polystyrene) subjected to uniaxial extension. In addition, we have characterized the equilibrium TC of polyethylene melts with average chain length ranging from $N=12$ to 5000 carbon atoms. Additional details on these systems have been included in Tables $\mathrm{S} 1$ and S2 in the Supporting Information. The preparation of the initial configurations is explained next, followed by a description of the additional equilibration, stretching, and short-term relaxation steps in our simulations. Finally, a brief analysis of how the thermal conductivity is calculated using the Green-Kubo method is given. The additional equilibration steps, NEMD stretching, and EMD measurement of TC and stress are all performed using the LAMMPS MD package. ${ }^{43}$

Initial Configurations. First, we consider a polyethylene melt consisting of 8 chains of 1000 carbon atoms each $($ PE8 $\times$ 1000 ). Note that for $N=1000$, or equivalently $M=14000 \mathrm{~g} /$ mol, the chain length is well above the entanglement chain length $\left(N_{\mathrm{e}} \simeq 75\right)$ or molecular weight $\left(M_{\mathrm{e}} \simeq 1050 \mathrm{~g} / \mathrm{mol}\right)$ given by simulation ${ }^{44}$ or by experiments $\left(N_{\mathrm{e}} \simeq 82, M_{\mathrm{e}} \simeq 1150\right.$ $\mathrm{g} / \mathrm{mol}) .^{45}$ The interatomic interactions for both molecular dynamics and Monte Carlo simulations are described with the transferable potentials for phase equilibria force field for united atoms (TraPPE-UA), in which the hydrogen atoms are lumped with the skeletal carbon atoms and the interactions between UAs are described collectively. ${ }^{46-48}$ Equilibration is achieved using the connectivity-altering Monte Carlo (CAMC) method. $^{41,49,50}$ For the equilibration of PE $8 \times 1000$, the following mix of moves was used: reptation (5\%), flip (10\%), rebridging (10\%), end-bridging (60\%), and end-regrowth with configurational bias (15\%). ${ }^{51}$ A detailed description of each $\mathrm{MC}$ move can be found elsewhere. ${ }^{52,53}$ CAMC has been shown to produce well-equilibrated molecular structural conformations, thermodynamics, entanglement density, as well as other key properties of polyethylene melts. ${ }^{54,55}$

During the equilibration of the samples used to test the TC dependence on molecular weight (listed in Table S2), additional connectivity-altering moves able to change the length of the chains were allowed (e.g., starting from a system of 10 chains of 1000 monomers each, equilibration results in a uniform distribution of chains $(500 \leq N \leq 1500)$ with average chain length 1000 and polydispersity index (PDI) $\leq 1.08$ ). Allowing a small degree of polydispersity has been shown to significantly speed up the equilibration of melts using CAMC. ${ }^{50}$ The following mix of moves was used for the systems summarized in Table S2 in the Supporting Information: reptation (10\%), rotation (5\%), flip (5\%), concerted rotation (30\%), end-bridging (15\%), doublebridging (15\%), intramolecular-double-bridging (10\%), selfend-bridging (9\%), and volume fluctuations (1\%). Note that no significant changes in thermal conductivity are observed between the monodisperse $\left(k_{\mathrm{eq}}(\mathrm{PE} 8 \times 1000)=0.27 \pm 0.03\right.$ $\mathrm{W} / \mathrm{m} \mathrm{K})$ and polydisperse $\left(k_{\text {eq }}(\mathrm{PE} 10 \times 1000)=0.28 \pm 0.02\right.$ $\mathrm{W} / \mathrm{m} \mathrm{K}$ ) systems (both shown in Figure 8 ).

Due to the molecular complexity of PS, production of wellequilibrated PS melts composed of long chains (i.e., $M \gtrsim M_{c}$ ) is significantly more demanding computationally and requires employing coarse-graining methods. ${ }^{42,56}$ In this study, monodisperse melts of atactic polystyrene (aPS) chains were generated and simulated following the procedure described by Vogiatzis and Theodorou. ${ }^{42}$ The monodisperse specimens contain 50\% meso-dyads obeying Bernoullian statistics and a chain length of 300 repeat units (coarse-grained sites). Given that $N_{\mathrm{e}} \simeq 125$ repeat units, ${ }^{57}$ this monodisperse melt is slightly above the entanglement limit $N_{\mathrm{c}}=2 N_{\mathrm{e}} \simeq 250 .{ }^{58}$ Bearing in 
mind the limitations of the atomistic MD simulations, the main part of this work is based on systems composed of 10 chains $($ PS10 $\times 300)$. As in previous works, ${ }^{42,59}$ we addressed the challenge of producing realistic aPS melts by employing two interconnected levels of representation: a coarse-grained level where equilibration is achieved through $\mathrm{CAMC}^{57}$ and an atomistic level that is recovered through reverse mapping. The atomistically detailed configurations obtained by reverse mapping are described by a united-atom model without partial charges, based on the work of Lyulin and Michels. ${ }^{60}$ Despite the fact that hydrogen atoms are not included in the description, the model can fully capture the structure, dynamics, and mechanical properties of aPS. ${ }^{42,57,61}$

Additional Equilibration Steps. Regarding the PE, after energy minimization with the Hessian-free truncated Newton algorithm, systems are subjected to the following equilibration process at $450 \mathrm{~K}$. Initially, an NVT temperature equilibration is performed for $1 \mathrm{~ns}$ using the velocity-rescale thermostat (rescaled 100 steps with a $5 \mathrm{~K}$ temperature window and a scale fraction of 0.5$)$. Then, the system is equilibrated in the canonical ensemble $(N V T)$ for $1 \mathrm{~ns}$; a pressure equilibration follows in the isothermal-isobaric ensemble (NPT) at 1 bar. Unless stated otherwise, the Nose-Hoover ${ }^{62,63}$ thermostat (barostat) is incorporated for maintaining the temperature (pressure) in conjunction with the MTK correction ${ }^{64}$ with an effective relaxation time of 0.1 (1.0) ps. After minimization of the potential energy at $500 \mathrm{~K}^{42}$ the PS10 $\times 300$ system is brought to a temperature of $450 \mathrm{~K}$ over $10 \mathrm{~ns}$ at constant pressure. Once the melt is at $450 \mathrm{~K}$, the equilibration is extended over $100 \mathrm{~ns}$ at constant pressure (NPT), during which the density of the melt is tracked to ensure that it remains constant.

Stretching and Short-Term Stress Relaxation. Once equilibrated, in a first step, both melts are subjected to NEMD uniaxial extension at constant pressure along the transverse directions $\left(N \dot{\varepsilon}_{x x} \sigma_{y y} \sigma_{z z} T\right)$. At specific times during the stretching, molecular configurations are stored at states of deformation given by the stretch ratio $\lambda=L_{x} / L_{0}$, where $L_{x}$ is the system box size in the direction of stretching and $L_{0}$ is the ensemble averaged length of the original cubic system. The stored deformed configurations are employed in a second step for the simultaneous determination of stress and TC. This second step consists of a 5 ns poststretching isochoric relaxation during which the strain remains constant (NVT ensemble). The stress per atom is calculated and averaged over the system to obtain the stress tensor. An average of the relevant stress tensor components is then computed using the last $0.5 \mathrm{~ns}$ of the poststretching isochoric relaxation step. Figure 1A presents the stress $\sigma=\tau_{11}-\left(\tau_{22}+\tau_{33}\right) / 2$ as a function of time during the stretching (green solid line) of PE8 $\times 1000$ at the highest used strain rate $\dot{\varepsilon}=7 \times 10^{8} \mathrm{~s}^{-1}$ (or Wi $=$ 3500). An example of the raw stress data during the poststretching isochoric relaxation step of a conformation extracted at the highest stretch ratio $\lambda \simeq 4$ is also shown in Figure 1A (gray translucent line). For clarity, in Figure 1A, we only show the smoothed stress relaxation data of a few deformed conformations (green dashed lines) obtained using a moving average Savitzky-Golay filter. ${ }^{65}$ For each of the extracted sample configurations, the last $0.5 \mathrm{~ns}$ of the poststretching isochoric relaxation is used to obtain a stress average, which we will refer to as the "relaxed stress". Note that even for the most extreme case (i.e., the highest strain and strain rate), the stress appears to reach a plateau by the end of the 5 ns run.

Throughout the text, we use the Weissenberg number (Wi) to compare the rheological behavior of polymers with different molecular weights (quantitatively) or even chemical constitutions (qualitatively). This dimensionless number $\mathrm{Wi}=\dot{\varepsilon} \tau_{\mathrm{m}}$ marks the transition between linear $(\mathrm{Wi}<1)$ and nonlinear $(\mathrm{Wi}>1)$ rheology and is defined as the product of the deformation rate $\dot{\varepsilon}=\frac{1}{L_{0}} \frac{\mathrm{d} L}{\mathrm{~d} t}$ and the stress mean relaxation time $\tau_{\mathrm{m}}=1 / 3 \quad \tau_{\mathrm{d}}$, which we have taken to be one-third of the reptation or disengagement time $\tau_{\mathrm{d}}$ to include the effect of constraint release. ${ }^{66}$ According to the comparison of the tube model and experimental evidence from linear melts, we have taken the simplified approximation $\tau_{\mathrm{d}} \sim 3 Z \tau_{\mathrm{R}} \cdot{ }^{58,66}$ Here, $Z=$ $N / N_{\mathrm{e}}$ is the entanglement number and $\tau_{\mathrm{R}}$ is the Rouse time. For PE $8 \times 1000$, we compute the Rouse time based on an estimate of the disengagement time $\tau_{\mathrm{d}}(\mathrm{C} 1000) \simeq 5000 \mathrm{~ns}$ (or $\tau_{\mathrm{m}} \simeq 1500 \mathrm{~ns}$ ) provided by Harmandaris et al., ${ }^{67}$ which results in a Rouse time $\tau_{\mathrm{R}}(\mathrm{C} 1000) \simeq 400 \mathrm{~ns}$. Since estimation of the Rouse time from molecular dynamics for a PS melt like ours remains a challenge, we opt to estimate the Rouse time $\tau_{\mathrm{R}}(\mathrm{PS} 10 \times 300) \simeq 3.8 \times 10^{-4} \mathrm{~s}\left(\right.$ or $\left.\tau_{\mathrm{m}} \simeq 8.2 \times 10^{-4} \mathrm{~s}\right)$ using the well-known empirical relation ${ }^{68}$

$$
\tau_{\mathrm{R}} \simeq \frac{12 M \eta_{0}}{\pi^{2} \rho R T}\left(\frac{M_{\mathrm{c}}}{M}\right)^{2.4}
$$

where the zero shear viscosity $\eta_{0}$ as a function of the molecular weight $M$ is taken from the experimental relation $\log \eta_{0}=$ $3.32 \log M-12.34^{69}$ at $183{ }^{\circ} \mathrm{C}$ and the Arrhenius relation for the zero shear viscosity $\eta_{0}(T)=\eta_{0}\left(T_{\text {ref }}\right) \exp \left[E^{*}(1 / R T-1 /\right.$ $\left.\left.R T_{\text {ref }}\right)\right]$ with $E^{*}=23.5 \mathrm{kcal} / \mathrm{mol}$, to obtain $\eta_{0} \sim 63 \mathrm{~Pa} \mathrm{~s}$ at 450 $\mathrm{K}^{70}$ In eq 2 , we have considered the critical entanglement molecular weight for polystyrene, $M_{c}=2 M_{e}=26000 \mathrm{~g} / \mathrm{mol}^{57}$ and a constant density value $\rho=0.89 \mathrm{~g} / \mathrm{cm}^{3}$ according to our simulation results (see inset to Figure $3 \mathrm{~B}$ ).

Proper evaluation and analysis of the stress in stretched samples are fundamental to testing the STR and require further discussion. Ideally, the evaluation should be performed on a time scale during which both the stress and TC tensors can be considered constant. That means that the stress determination needs to be made in a short time period so that the stretched system can be considered as "quenched" from the point of view of the entanglement network in the melt. In addition, the evaluation of the stress should be achieved simultaneously with the measurement of TC, so that it corresponds to the same state of the system. Furthermore, if we are to compare simulation and experimental results, it is desirable that any contributions to the stress due to fast deformation rates required by NEMD are relaxed. The methodology followed for the determination of the stress in our study (exemplified in Figures $1 \mathrm{~A}$ and $3 \mathrm{~A}$ ) intends to satisfy all of these conditions.

In Figure 1B, we show a comparison of the stretching (solid translucent lines without symbols) and average relaxed (cross symbols joined by straight lines) stress-strain responses of the $\mathrm{PE}$ system as a function of the stretch ratio $\lambda$. As can be seen in both Figure $1 \mathrm{~A}$ and $\mathrm{B}$, a significant amount of stress relaxation takes place in the first couple of nanoseconds of each poststretching isochoric relaxation. We emphasize that the discrepancy between the stress during stretching and the relaxed stress becomes smaller as the deformation rate decreases and is negligible at the lowest strain rate (see Figure $1 \mathrm{~B}$, where stretching stress and relaxed stress superimpose for 
the lowest deformation rate at $\mathrm{Wi}=50(\mathrm{red}))$. Similarly, it is apparent in Figure $1 \mathrm{~B}$ that for low strains $(\lambda \lesssim 2.0)$, the stress relaxation is rather small (i.e., when the system is subjected to small strains, the deformation results in a configuration already displaying this pseudoequilibrium relaxed stress).

We argue that the methodology proposed for the estimation of the stress is possible due to the separation of time scales for the different relaxation processes in play. We consider the durations of stretching and poststretching isochoric relaxation steps (Figures $1 \mathrm{~A}$ and $3 \mathrm{~A}$ ) and compare them to the polymer relaxation time constants following deformation, which are strongly dependent on the chemical constitution and molecular weight of each system (See Table 1). The complex

Table 1. Characteristic Relaxation Times ${ }^{68,74}$ and Entanglement Plateau Moduli ${ }^{75}\left(G_{\mathrm{N}}\right)$ : Local Segmental Relaxation Time $\left(\tau_{0}\right)$, Entangled Strand Relaxation Time $\left(\tau_{\mathrm{e}}\right)$, Rouse Time $\left(\tau_{\mathrm{R}}\right)$, and Disengagement Time $\left(\tau_{\mathrm{d}}\right)$

$\begin{array}{cllrrc}\text { polymer } & \tau_{0}(\mathrm{~ns}) & \tau_{\mathrm{e}}(\mathrm{ns}) & \tau_{\mathrm{R}}(\mathrm{ns}) & \tau_{\mathrm{d}}(\mathrm{ns}) & G_{\mathrm{N}}(\mathrm{MPa}) \\ \text { PE } 8 \times 1000 & 0.0005 & 2.6 & 400 & 5000 & 2.1 \\ \text { PS10 } \times 300 & 4.2 & 66000 & 380000 & 900000 & 0.2\end{array}$

dynamics taking place during deformation of an entangled polymeric melt can be described in terms of a number of processes characterized by different time scales. ${ }^{58}$ As a first approximation to the problem, we consider four different processes/time scales: the local segmental relaxation given by $\tau_{0}$; the entangled strand relaxation through Rouse motion at $\tau_{\mathrm{e}}$ $\sim N_{\mathrm{e}}{ }^{2} \tau_{0}$; the contour length relaxation through primitive path retraction (e.g., segments remaining oriented) at times of order $\tau_{\mathrm{R}} \sim N^{2} \tau_{0}$; and, finally, the relaxation of the strands' orientation via reptation at times $\tau_{\mathrm{d}} \sim\left(N^{3} / N_{\mathrm{e}}\right) \tau_{0}$ (although both simulation and experiments have shown $\left.{ }^{58,71} \tau_{\mathrm{d}} \propto N^{3.4}\right)$. Table 1 summarizes these characteristic relaxation times obtained from the relationships above, using our estimate for the Rouse times for PE $8 \times 1000$ and PS10 $\times 300$. We find the estimated values presented in Table 1 to be in good agreement with experimental and simulation results. ${ }^{72,73}$ Examination of these relevant time scales clearly shows that neither $\tau_{\mathrm{R}}$ nor $\tau_{\mathrm{d}}$ time scales are accessed in our simulations. It would seem possible that the relaxation associated with $\tau_{\mathrm{e}}$ could explain the relaxation of the stress observed in Figure $1 \mathrm{~A}$ for the polyethylene system. However, for polystyrene, $\tau_{\mathrm{e}}$ is much larger than the simulation time during the poststretching isochoric relaxation presented in Figure $3 \mathrm{~A}$, and we do observe the same short relaxation of the stress in this system.

To better understand the difference between the relaxed stress and stress achieved during stretching, we looked at the local structure during the stretching and subsequent poststretching isochoric relaxation steps. In Figure 2, we display the probability distributions of bond lengths $l$, bond angles $\theta$, and torsional angles $\varphi$ before (black), at the end of stretching (red), and after the subsequent relaxation (dashed green) of PE8 $\times 1000$ for the most extreme case studied $(\dot{\varepsilon}=7$ $\times 10^{8} \mathrm{~s}^{-1}$ and $\lambda \simeq 4$ ). We observe no significant deviations from equilibrium during the stretching (or relaxation) process for the bond length $P(l)$ and bond angle $P(\theta)$ probability distributions (see insets to Figure 2). On the other hand, the probability distribution of torsional angles $P(\varphi)$ in Figure 2 departs from equilibrium during stretching toward configurations that are richer in antiperiplanar (ap, trans) conformations. During the $5 \mathrm{~ns}$ poststretching isochoric

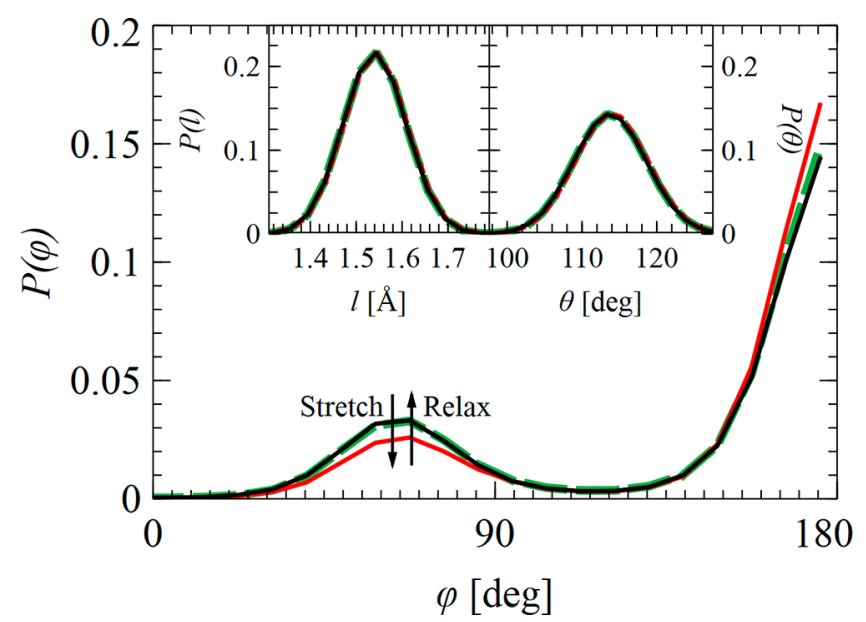

Figure 2. Torsional angle $\varphi$ distribution for PE $8 \times 1000$ in different states. During the stretching, the system goes from a well-equilibrated conformation before any deformation (black solid line) to the highest deformation $\lambda=4$ achieved at $\mathrm{Wi}=3500$ (red solid line). The stretched system undergoes subsequent relaxation of the stress in the $N V T$ ensemble during poststretching isochoric relaxation to recover after $5 \mathrm{~ns}$ essentially the same original distribution of torsional angles (green dashed line). The insets show how the bond length $l$ and angle $\theta$ distributions remain undisturbed over the same process.

relaxation step, segmental diffusion leads the overstretched configurations to an equilibrium-like distribution of torsional angles comparable to that of the unstretched system (i.e., the equilibrium torsion angle distribution is recovered by overcoming barriers associated with cooperative torsional transitions associated with segmental relaxation). Recent simulation work by $\mathrm{Hsu}$ et al. ${ }^{71,76}$ using primitive path analysis on highly strained entangled melts has shown a similarly fast short-range relaxation (i.e., chains quickly relax inside the tube, without significant relaxation of the tube conformation). Based on this observation and the time constants in Table 1, we argue that the orientation of chain strands between entanglements is not significantly relaxed in the time scale of the poststretching isochoric step in our simulations. However, we highlight that the small variation in the torsional angle distribution displayed in Figure 2 results in a significant increase in the perceived stress in the system. For example, at the highest strain rate (Wi $=3500)$, the maximum stress during stretching $\left(\sigma_{\mathrm{str}}^{\max }=114 \pm\right.$ $20 \mathrm{MPa}$ ) is about 5 times that of the system once the torsional angle distribution has relaxed $\left(\sigma_{\mathrm{rel}}^{\max }=18.4 \pm 1.9 \mathrm{MPa}\right)$, while the latter clearly remains significantly far from the unstretched equilibrium stress (i.e., zero stress). Our interpretation is that the stress buildup due to this shift in the torsional angles does not correspond to the stress held by the molecular entanglement network, which is comparable to the stress observed in experiments, but is the result of the fast strain rates imposed during NEMD stretching of the system. The substantial difference in time scales between the relaxation processes allows us to measure the stress of the system after the local conformation has been recovered (i.e., torsional angle redistribution in $1-5 \mathrm{~ns}$ with a stress relaxation in $t>\tau_{0}$ and polymer entanglement network relaxation not present at times $\left.t \ll \tau_{\mathrm{R}} \sim 400 \mathrm{~ns}\right)$. Following this conjecture, we used the method described in Figure 1A to obtain the relaxed stress of all deformed configurations. Note that no significant changes in the density of the system are induced by the deformation process (see inset to Figure 1B). A clearer representation of the 

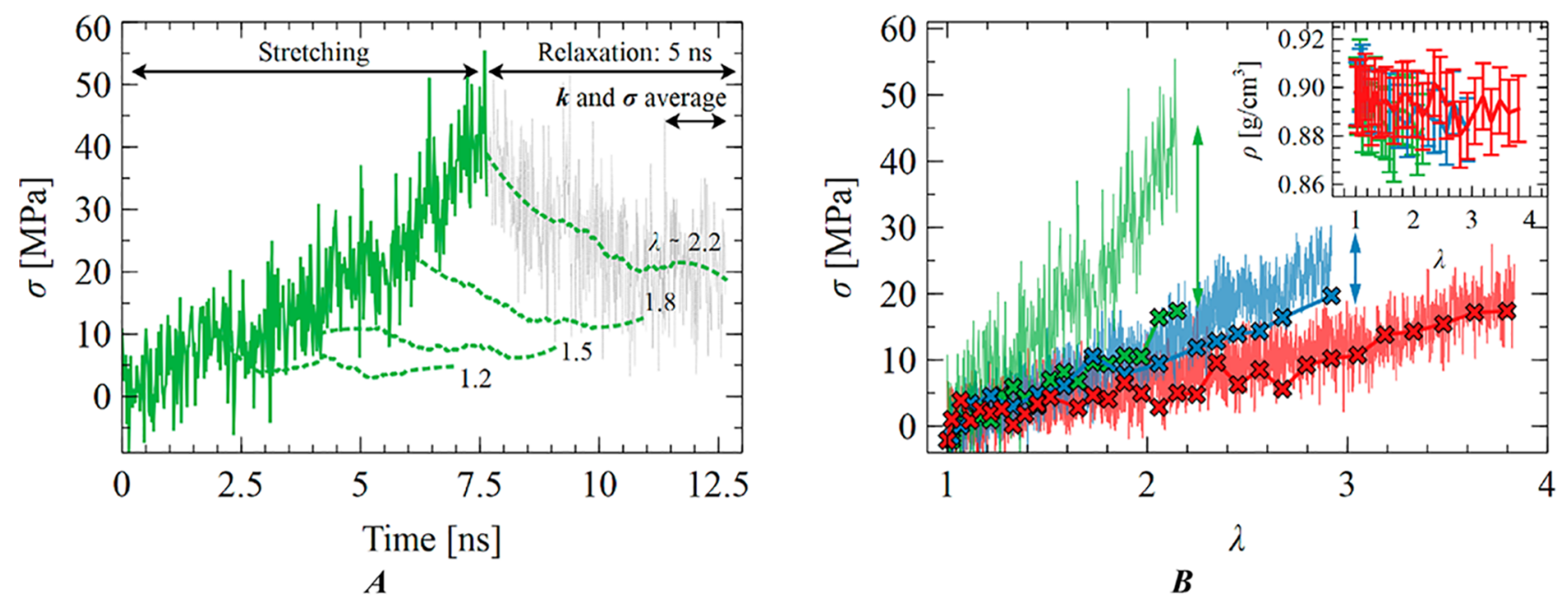

Figure 3. Stress determination approach for PS10 $\times 300$. (A) The green solid line represents the stress as a function of time during NEMD stretching for the melt at the highest $\mathrm{Wi}=47000$. The green dashed lines display the moving averages of the subsequent stress relaxation for a selection of configurations with stretch ratios $\lambda \simeq 1.2,1.5,1.8$, and 2.2 (from bottom to top). The gray solid line shows the raw stress data without averaging. (B) Comparison of the stress measured during NEMD stretching (solid lines) and the relaxed stress averaged over the last $0.5 \mathrm{~ns}$ of $5 \mathrm{~ns}$ poststretching isochoric relaxation (cross symbols) at $\mathrm{Wi}=2400$ (red), $\mathrm{Wi}=4700$ (blue), and $\mathrm{Wi}=47000$ (green). The inset shows that the variations in the density of samples are within the numerical errors. All simulations were performed at $450 \mathrm{~K}$.

stress-strain behavior (i.e., showing only the relaxed stress as a function of the stretch ratio) is displayed in Figure 4B.

To determine the stress of the deformed PS10 $\times 300$ configurations, we followed the same approach as for PE8 $\times$ 1000. Figure $3 \mathrm{~A}$ shows the highest deformation rate $(\mathrm{Wi}=$ $47000)$ stretching and stress relaxation results. Note that in Figure $3 \mathrm{~A}$ we observe the same qualitative behavior with respect to strain and strain rate as in Figure 1A (i.e., higher strain results in a higher difference between the stress before and after the poststretching isochoric stress relaxation). For PS, as one might expect given the estimate for $\tau_{0}$ in Table 1 , we observe that the relaxation of the stress during the poststretching isochoric relaxation step is somewhat slower. Figure $3 \mathrm{~B}$ shows the comparison of stress during stretching and the "relaxed" stress as a function of the stretch ratio. As for the polyethylene system, we do not observe significant changes in density induced by the deformation process (see inset to Figure 3B). As for polyethylene, when Wi decreases (i.e., for low strain rates), the difference between the stress during stretching and the relaxed stress is very small (Figure 3B). The apparently higher uncertainty displayed in the stress results for PS10 $\times 300$ partially results from the lower absolute values of stress studied for this material (i.e., $\sim 150 \mathrm{MPa}$ for PE and $\sim 55$ MPa for PS).

It is worthwhile discussing the dependence of the relaxed stress on the strain rate that is observed in both Figures $1 \mathrm{~B}$ and $3 \mathrm{~B}$. Table 2 summarizes the range of strain rates employed in our simulations and its relative magnitude compared to the inverse of the Rouse and entanglement times. As indicated by Hsu et al., ${ }^{76}$ having $\tau_{\mathrm{R}}^{-1}<\dot{\varepsilon}<\tau_{\mathrm{e}}^{-1}$ will allow certain relaxation during deformation and therefore explain the stress depend-

Table 2. Stretching Conditions

\begin{tabular}{|c|c|c|c|c|c|c|}
\hline \multirow{2}{*}{$\frac{\text { polymer }}{\dot{\varepsilon}\left(\mathrm{s}^{-1}\right)}$} & \multicolumn{3}{|c|}{ PE $8 \times 1000$} & \multicolumn{3}{|c|}{ PS $10 \times 300$} \\
\hline & $1 \times 10^{7}$ & $1 \times 10^{8}$ & $7 \times 10^{8}$ & $5 \times 10^{6}$ & $1 \times 10^{7}$ & $1 \times 10^{8}$ \\
\hline$\dot{\varepsilon} \tau_{\mathrm{R}}$ & 4.0 & 40 & 280 & 1900 & 3800 & 38000 \\
\hline$\dot{\varepsilon} \tau_{\mathrm{e}}$ & 0.026 & 0.26 & 1.82 & 330 & 660 & 6600 \\
\hline
\end{tabular}

ence on the strain rate observed in Figure $1 \mathrm{~B}$ for the PE system. However, our estimation of the characteristic relaxation times for polystyrene would indicate that the same argument cannot be made for the results in Figure 3B (notwithstanding the same qualitative behavior observed for the two materials). As a result of the significantly higher relaxation times found for PS, one would expect maximum orientation of the primitive path segments to be achieved by essentially affine deformation $\left(\dot{\varepsilon} \gg 1 / \tau_{\mathrm{e}}\right)$ at all of the strain rates applied, which should result in no strain rate dependence. We note that for the lowest strain rate (or $\mathrm{Wi}=2400$ ) employed for the PS system, it requires approximately $300 \mathrm{~ns}$ to reach the maximum deformation $\lambda \simeq 4$. Our results could indicate that entangled strand relaxation through the Rouse motion process is active at time scales shorter than our estimate for $\tau_{\mathrm{e}}$.

Determination of Thermal Conductivity (TC). The thermal conductivity of each stretched molecular configuration is obtained from the autocorrelation function of the microscopic heat flux components $J_{\alpha}$ in various directions $\alpha$ using EMD and the Green-Kubo $(\mathrm{G}-\mathrm{K}) \operatorname{method}^{77}$ in the $5 \mathrm{~ns}$ poststretching isochoric relaxation.

$$
k_{\alpha \beta}=\frac{V}{k_{\mathrm{B}} T^{2}} \int_{0}^{\infty} J_{\alpha}(0) J_{\beta}(t) \mathrm{d} t
$$

where $V$ is the system volume, $k_{\mathrm{B}}$ is the Boltzmann constant, and $T$ is the temperature. The microscopic heat flux vector is defined as

$$
\mathbf{J}=\frac{1}{V} \frac{\mathrm{d}}{\mathrm{d} t} \sum_{i=1}^{N} \varepsilon_{i} \mathbf{r}_{i}
$$

where $\varepsilon_{i}$ is the energy carried by each one of the $N$ particles at $\mathbf{r}_{i}$ moving with instantaneous velocity $\mathbf{v}_{i}$ and is given by 


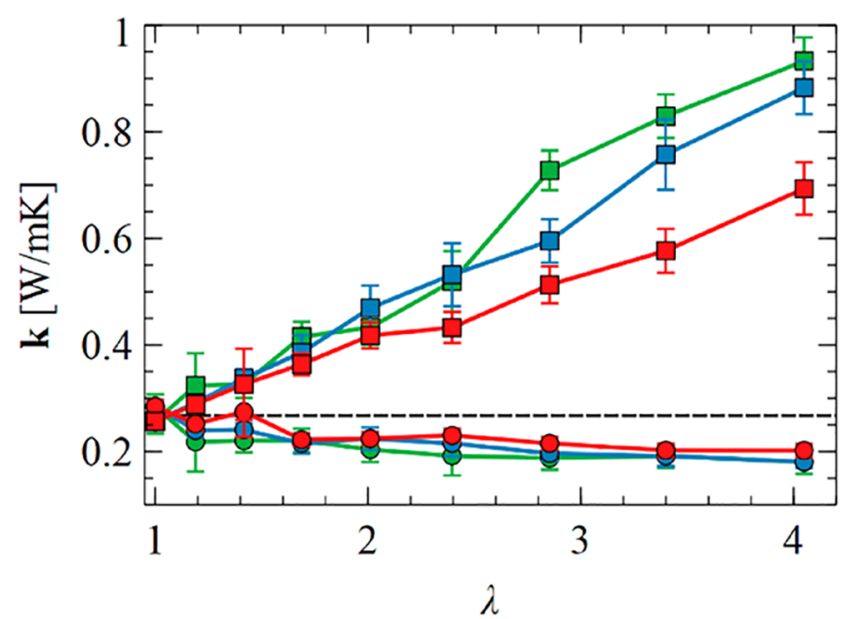

$\mathbf{A}$

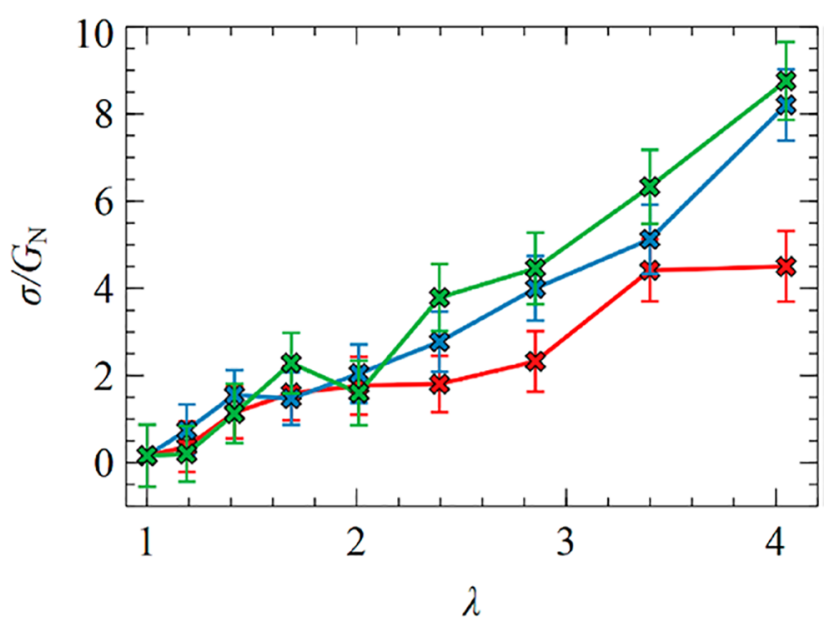

B

Figure 4. TC and relaxed stress-strain behavior of PE $8 \times 1000\left(\mathrm{Wi}=50\left(\right.\right.$ red), $\mathrm{Wi}=500$ (blue), and Wi $=3500$ (green)). (A) $k_{11}($ squares $)$ and $k_{22}$ (circles) as a function of the stretch ratio $\lambda$. The dashed black line represents the equilibrium value for the TC of the unstretched sample $k_{\text {eq }}^{\text {sim }}$ $(\mathrm{PE} 8 \times 1000)=0.27 \pm 0.03 \mathrm{~W} / \mathrm{m} \mathrm{K}$. (B) Relaxed stress normalized by the entanglement plateau modulus $G_{\mathrm{N}}=2.1 \mathrm{MPa}^{75}$ as a function of the stretch.

$$
\begin{aligned}
\varepsilon_{i} & =\frac{\mathbf{p}_{i}^{2}}{2 m_{i}}+\mathcal{V}_{i}=\frac{\mathbf{p}_{i}^{2}}{2 m_{i}}+\frac{1}{2} \sum_{\substack{j=1 \\
j \neq i}}^{N} u^{(2)}\left(\left\{\mathbf{r}_{i}, \mathbf{r}_{j}\right\}\right) \\
& +\frac{1}{3} \sum_{\substack{j=1 \\
j \neq i}}^{N} \sum_{\substack{k=1 \\
k \neq i, j}}^{N} u^{(3)}\left(\left\{\mathbf{r}_{i}, \mathbf{r}_{j}, \mathbf{r}_{k}\right\}\right) \\
& +\frac{1}{4} \sum_{\substack{j=1 \\
j \neq i}}^{N} \sum_{\substack{k=1 \\
k \neq i, j}}^{N} \sum_{\substack{l=1 \\
l \neq i, j, k}}^{N} u^{(4)}\left(\left\{\mathbf{r}_{i}, \mathbf{r}_{j}, \mathbf{r}_{k}, \mathbf{r}_{l}\right\}\right)
\end{aligned}
$$

In eq $5, m_{i}$ and $p_{i}$ are the mass and momentum of particle $i$, respectively, and $\mathcal{V}_{i}$ is the contribution to the system potential energy attributed to particle $i$. The quantities $u^{(2)}, u^{(3)}$, and $u^{(4)}$ are potential energy terms contributed by pairs (nonbonded interactions and bond stretching potentials), triplets (bond angle bending potentials), and quadruplets (torsional and improper torsional potentials) of particles, respectively. In the expression for the total potential energy, there is at most one $u^{(2)}$ term involving a particular pair of particles $i$ and $j$, at most one $u^{(3)}$ term involving a particular triplet of particles $i, j$, and $k$, and so on. To emphasize that the terms depend on which particles are involved, but not on the order in which these particles are taken, the arguments of the $u$ 's in eq 5 above have been placed within curly brackets. Using the chain rule, eq 4 is typically split into three contributions: (1) the kinetic energy heat flux $\mathbf{J}_{\text {kin }}$, (2) the potential energy heat flux $\mathbf{J}_{\text {pot }}$ and (3) the virial stress heat flux $\mathbf{J}_{\text {stress. }}$. The first two correspond to the convection of energy carried by the particles, namely

$$
\begin{aligned}
& \mathbf{J}_{\text {kin }}+\mathbf{J}_{\text {pot }}=\sum_{i=1}^{N} \varepsilon_{i} \mathbf{v}_{i} \\
& \mathbf{J}_{\text {stress }}=\sum_{i=1}^{N} \frac{\mathrm{d} \varepsilon_{i}}{\mathrm{~d} t} \mathbf{r}_{i}
\end{aligned}
$$

The microscopic heat flux vector can be shown as ${ }^{78}$

$$
\mathbf{J}=\frac{1}{V} \sum_{i=1}^{N}\left[\left(\mathcal{V}_{i}+\frac{\mathbf{p}_{i}^{2}}{2 m_{i}}\right) \mathbf{v}_{i}-\sum_{\substack{j=1 \\ j \neq i}}^{N}\left(\frac{\partial \mathcal{V}_{j}}{\partial \mathbf{r}_{i}} \cdot \mathbf{v}_{i}\right)\left(\mathbf{r}_{i}-\mathbf{r}_{j}\right)\right]
$$

Additional details on the implementation of the G-K method, the convergence of both the integral in eq 3 and the integrand over the 2000 fs integration periods, and the invariance of the heat flux with respect to rigid translation of the coordinate system are provided in the Supporting Information.

The Results section can be divided into two parts. In the first part, we study the anisotropy in TC and its relation to stress in melts subjected to uniaxial extension. For this part, only the diagonal components of the tensor $k_{\alpha \alpha}$ are relevant, and since the samples were stretched in the 1-direction, we take $k_{22} \cong k_{33}$ as a result of symmetry. An average of the relevant TC components is obtained simultaneously with the averaged relaxed stress during the last $0.5 \mathrm{~ns}$ of each $5 \mathrm{~ns}$ poststretching isochoric relaxation. In the second part, we test the hypothesis of preferential heat transport along the chain backbone by studying a number of melts with a wide range of average chain lengths. For this second part, we only determine the equilibrium (i.e., unstretched) system $\mathrm{TC}$, which is defined as $k_{\mathrm{eq}}=1 / 3 \operatorname{tr}\left(\mathbf{k}_{\mathrm{eq}}\right)$.

\section{RESULTS}

As described in the Simulation Methods section, during poststretching isochoric relaxation, the TC tensor components are obtained simultaneously with the stress. The results for $k_{11}$ and $k_{22}$ as a function of the stretch ratio $\lambda$ for PE $8 \times 1000$ are presented in Figure 4A. We observe an increase in TC in the direction parallel to the stretch and a decrease in the perpendicular direction with respect to the equilibrium values for the TC of the unstretched system (dashed black line in Figure 4A). For polyethylene, the range of experimental values for equilibrium thermal conductivity in the literature is quite wide, $k_{\mathrm{eq}}^{\exp }(\mathrm{PE}) \sim 0.2-0.4 \mathrm{~W} / \mathrm{m} \mathrm{K}^{79}$ We suspect that the wide range of results is because of varying degrees of crystallinity in the studied samples as well as the large uncertainty between measurement methods and samples. However, the value obtained from simulations $k_{\mathrm{eq}}^{\mathrm{sim}}(\mathrm{PE} 8 \times 1000)=0.27 \pm 0.03$ $\mathrm{W} / \mathrm{m} \mathrm{K}$ is well within the range offered in the literature. We observe qualitative agreement with experiments on the dependence of anisotropy on strain rate in melts (i.e., faster deformation leads to higher stress and therefore higher 
anisotropy). Note that, in contrast to the results by Liu et al., ${ }^{26}$ showing the opposite trend in anisotropy with respect to the strain rate in PE, we deform and study the TC of the samples above the glass-transition temperature. Using the results in Figure 1, we determine the relaxed stress-strain behavior of this system, which is shown in Figure 4B.

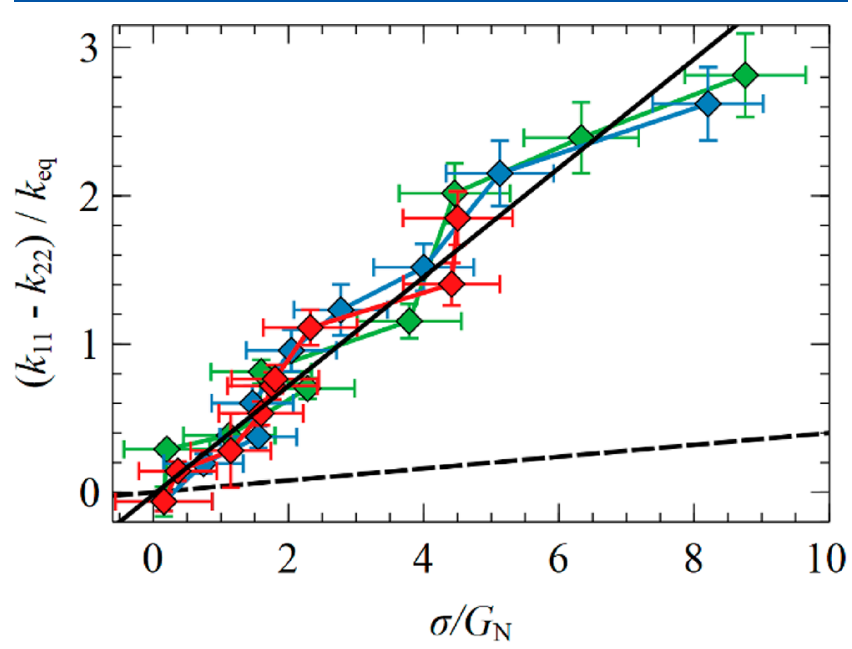

Figure 5. Test of the STR for PE8 $\times 1000$ using the results for TC and relaxed stress in Figure 4. As in previous figures showing results for PE $8 \times 1000$, the coloring corresponds to $\mathrm{Wi}=50$ (red), $\mathrm{Wi}=500$ (blue), and $\mathrm{Wi}=3500$ (green). The solid line represents a linear fit to the simulation data with $C_{t} G_{N}=0.36 \pm 0.03$, where the entanglement plateau modulus $G_{\mathrm{N}}=2.1 \mathrm{MPa}^{75}$ The dashed line is given by the universal value $\left(C_{\mathrm{t}} G_{\mathrm{N}}=0.04\right)$ found in experiments. ${ }^{27,28,35-38}$

In Figure 5, we have combined the TC and stress-strain behavior of Figure $4 \mathrm{~A}$ and $\mathrm{B}$ to test the STR, which for a system subjected to uniaxial extension can be expressed as

$$
\frac{k_{11}-k_{22}}{k_{\text {eq }}}=C_{\mathrm{t}} G_{\mathrm{N}} \frac{\sigma}{G_{\mathrm{N}}}
$$

where $C_{\mathrm{t}} G_{\mathrm{N}}$ is referred to as the normalized stress-thermal coefficient and $G_{\mathrm{N}}$ is the entanglement plateau modulus of the material, which for polyethylene is $2.1 \mathrm{MPa}^{75}$ As in Figures 1 and 4 , the coloring corresponds to $\mathrm{Wi}=50$ (red), $\mathrm{Wi}=500$ (blue), and $\mathrm{Wi}=3500$ (green). The solid line represents a linear fit to the data with $C_{t} G_{\mathrm{N}}=0.36 \pm 0.03$. To our knowledge, the data presented in Figure 5 represent the first molecular dynamics validation of the STR (i.e., a linear relationship between TC and stress tensors). The STRpredicted linear behavior holds over the whole range of stresses studied (up to $\sim 10 \times G_{\mathrm{N}}$ ). Following the approach in ref 80 , we have computed the birefringence $\Delta n$ (i.e., optical anisotropy) to test the stress-optic rule. Our results, included in the Supporting Information, suggest failure of the linear relationship between birefringence and stress for $\sigma / G_{\mathrm{N}} \lesssim 6$ and, therefore, support experimental evidence of an extended range of applications for the STR. Note that the same normalized stress-thermal coefficient applies independently of the strain rate, as in experimental evidence for PS and poly(methyl methacrylate) (PMMA) melts. ${ }^{27}$ This result is not trivial, since it further validates our approach to determining the stress in the stretched configurations. However, we find that the value for the normalized stress-thermal coefficient $C_{\mathrm{t}} G_{\mathrm{N}}=0.36 \pm 0.03$ (solid line in Figure 5) is an order of magnitude larger than the expected value $C_{t} G_{N} \sim 0.04$ (dashed line in Figure 5) based on experimental studies for PS, PMMA, and polydimethylsiloxane (PDMS) melts ${ }^{27,28,38}$ and lightly cross-linked elastomers. ${ }^{35-37}$ Note that a stronger dependence of TC on strain has been reported in vulcanized polyisoprene by Tautz et al. ${ }^{81}$ (experiments) and Engelmann et al. ${ }^{82}$ (simulation). Experimental validation of the STR for polyethylene remains a challenge due to its low glass-transition temperature and its affinity for crystallization. Nevertheless, our result for $C_{t} G_{N}$ is in direct contradiction with the universal

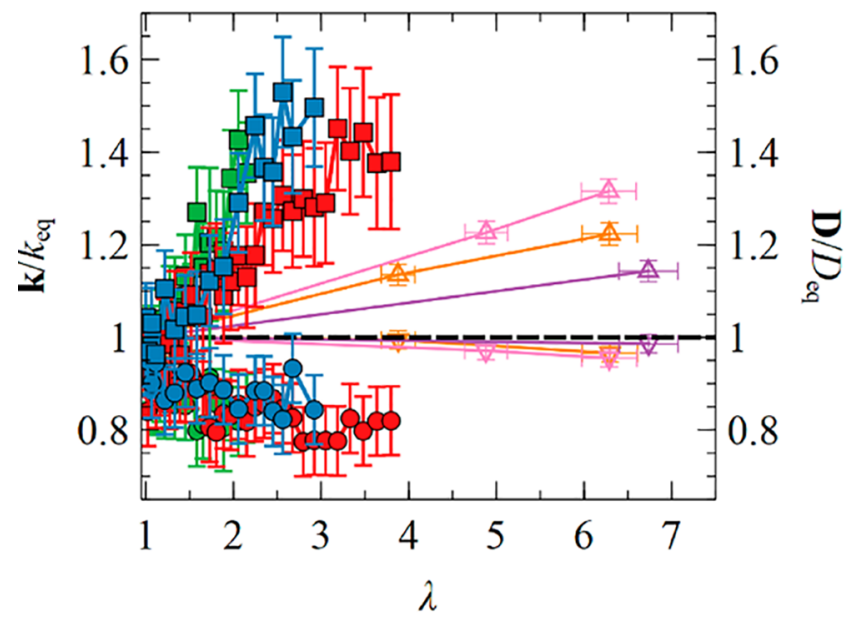

$\mathbf{A}$

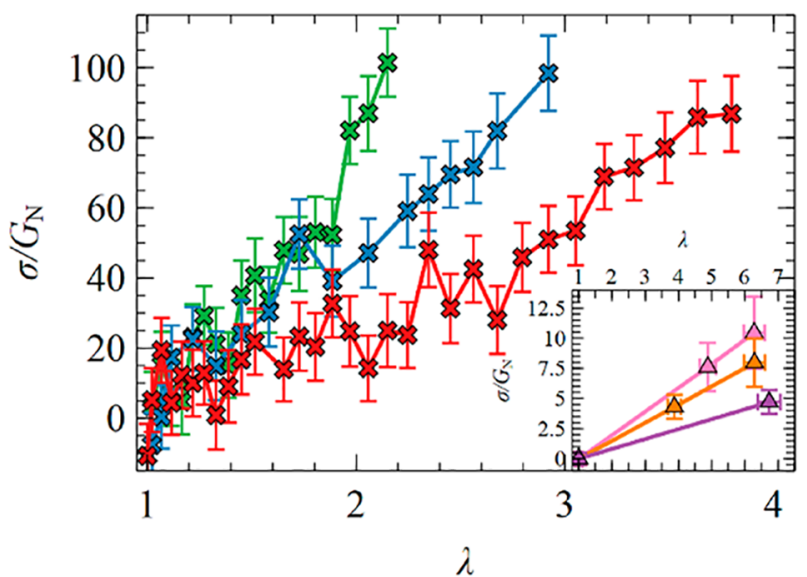

B

Figure 6. (A) PS10 $\times 300$ thermal conductivity tensor components $k_{11}$ (squares) and $k_{22}$ (circles) normalized by the equilibrium TC $\left(k_{\text {eq }}(\mathrm{PS} 10 \times\right.$ $300)=0.23 \pm 0.02 \mathrm{~W} / \mathrm{m} \mathrm{K}$, dashed line) as a function of the stretch ratio $\lambda$. The experimental thermal diffusivity measurements by Gupta et al. ${ }^{27}$ are shown as up and down triangles for $D_{11}$ and $D_{22}$, respectively, at $\mathrm{Wi}=30$ (purple), $\mathrm{Wi}=150$ (orange), and $\mathrm{Wi}=600$ (pink). (B) Relaxed stress of PS10 $\times 300$ melt stress normalized by $G_{\mathrm{N}}=0.2 \mathrm{MPa}^{75}$ as a function of the stretch ratio for $\mathrm{Wi}=2400$ (red), $\mathrm{Wi}=4700$ (blue), and Wi $=47000$ (green). The inset shows the experimental data from Gupta et al. ${ }^{27}$ at $\mathrm{Wi}=30$ (purple triangles), $\mathrm{Wi}=150$ (orange triangles), and $\mathrm{Wi}=600$ (pink triangles). 
(or chemical constitution independent) character of the STR suggested by experiments. ${ }^{27,28,35-38}$ We have considered size effects as a possible source of the discrepancy. However, analysis of a system with twice the number of chains and half the chain length produces essentially the same stress-thermal coefficient (see the Supporting Information Figure S9). Furthermore, an analysis of the ratio of the chain end-to-end vector (or the gyration radius) and the box length in the direction of stretching shows no additional stretching due to image-image interactions (see the Supporting Information Figure S10). Therefore, we conclude that system size effects are absent from our simulations.

Closer examination of the different polymer chemical constitutions studied by Venerus and co-workers ${ }^{27,28,35-38}$ shows that all of the systems studied present relatively low entanglement plateaus $G_{\mathrm{N}}$ ranging from 0.2 to $0.76 \mathrm{MPa}$ compared to polyethylene $G_{\mathrm{N}}=2.1 \mathrm{MPa},{ }^{75}$ which can be explained using topological analysis. ${ }^{44}$ This high entanglement plateau, which sets PE apart from other common melts, is due to its high entanglement density and chain stiffness (e.g., it has been shown that using tube models to reproduce the rheology of $\mathrm{PE}$ melts requires employing a semiflexible primitive path $^{83}$ ). Furthermore, we noticed that the two cross-linked polybutadiene systems ${ }^{35}$ with the highest entanglement plateau studied experimentally $\left(G_{\mathrm{N}}=0.760 \mathrm{MPa}\right)$ display the highest normalized stress-thermal coefficient $C_{t} G_{N} \sim 0.055$, although the range of variation is small. These results, together with the saturating behavior of thermal conductivity as a function of molecular weight around the entanglement limit (that will be discussed later in the paper), have led us to speculate that the large magnitude of the stress-thermal coefficient is related to the high entanglement plateau in PE melts.

To test this conjecture, we studied a polystyrene melt $\left(G_{\mathrm{N}}=\right.$ $\left.0.2 \mathrm{MPa}^{75}\right)$ consisting of 10 chains of 300 monomers each $($ PS10 $\times 300, M=31000 \mathrm{~g} / \mathrm{mol})$. For PS, we also find good agreement with typical equilibrium experimental values of the thermal conductivity, $k_{\mathrm{eq}}^{\exp }(\mathrm{PS}) \sim 0.18 \mathrm{~W} / \mathrm{m} \mathrm{K}^{84}$ while $k_{\mathrm{eq}}^{\text {sim }}$ $(\mathrm{PS} 10 \times 300)=0.23 \pm 0.04 \mathrm{~W} / \mathrm{m} \mathrm{K}$. Although the Rouse relaxation time for this polymer is much longer than for $\mathrm{PE}$, we were able to study a similar range of Wi because of the lower molecular weight with respect to the entanglement limit (i.e., lower $Z$ ). However, the range of strain rates and strains used resulted in significantly larger normalized stress values. The difference in range in the normalized stress $\sigma / G_{\mathrm{N}}$ results from the much lower plateau modulus of this material $G_{\mathrm{N}}=0.2$ $\mathrm{MPa}$, which gives rise to "relaxed stresses" of up to $100 \times G_{\mathrm{N}}$ as opposed to $10 \times G_{\mathrm{N}}$ for PE $8 \times 1000$. In fact, comparison of Figures $1 \mathrm{~A}$ and $3 \mathrm{~A}$ shows how the absolute highest stress studied for polyethylene is almost three times that of polystyrene.

In Figure $6 \mathrm{~A}$ and $\mathrm{B}$, we present our simulation results for the TC and stress of PS10 $\times 300$ as a function of stretch ratio $\lambda$ for Wi from 2400 to 47000 . As for PE $8 \times 1000$, we observe an increase (decrease) of TC in the direction parallel (perpendicular) to stretch as samples are deformed. For this melt, we observe a decrease in anisotropy in TC only at the lowest strain rate or $\mathrm{Wi}$. This effect indicates that some stress relaxation occurs during the longest stretching experiments used at the lowest rate (i.e., during the stretching, the entangled strand relaxation through the Rouse motion process is active). Furthermore, in the stress-strain results in Figure $6 \mathrm{~B}$, we observe how for large strains (stretch ratios $\lambda>1.5$ ) there is a clear dependence of stress on the strain rate. In both
Figure $6 \mathrm{~A}$ and the inset to $6 \mathrm{~B}$, we have included the experimental results by Gupta et al., ${ }^{27}$ characterizing the thermal transport and mechanical behavior of a polydisperse polystyrene system with $M=260000 \mathrm{~g} / \mathrm{mol}(N \sim 2500)$ subjected to uniaxial extension. For this system, the authors estimate a mean relaxation time $\tau_{\mathrm{m}} \sim 3000 \mathrm{~s}$, which for the strain rates studied results in $\mathrm{Wi}=30$ (purple triangles), $\mathrm{Wi}=$ 150 (orange triangles), and $\mathrm{Wi}=600$ (pink triangles). In their study, thermal transport measurements are made with the forced Rayleigh scattering (FRS) technique. FRS allows characterization of the thermal diffusivity (TD) tensor $\mathbf{D}=$ $\mathbf{k} / \rho \hat{\mathbf{c}}_{\mathrm{p}}$, where $\hat{\mathrm{c}}_{\mathrm{p}}$ is the specific heat at constant pressure. In Figure 6A, TD experimental results by Gupta et al. ${ }^{27}$ show the same qualitative behavior observed in our simulation results (i.e., increase of TC in the direction parallel to the stretch and decrease in the perpendicular direction, and higher Wi resulting in higher anisotropy). To facilitate the comparison with experiments, both $\mathrm{TC}$ and $\mathrm{TD}$ are normalized by the equilibrium values in Figure 6A. Note that TC (simulation) and TD (experimental) values have been included with two different axes in Figure 6A but with the same scale. Overall, the results from both experiments and simulations highlight that the dependence of anisotropy in TC on strain rate is more significant at lower Wi numbers or when significant relaxation of the orientation/stress can occur during stretching. A direct comparison of the stress-strain behavior, like the one presented in Figure 6A for TC, is not very illustrative given the different ranges of stretch ratios $(\lambda \sim 1-4$ in simulations and $\lambda \sim 4-7$ in experiments) and normalized stresses (up to $100 \times G_{\mathrm{N}}$ in simulations and up to $15 \times G_{\mathrm{N}}$ in experiments). Nonetheless, the experimental stress-strain results by Gupta et al. ${ }^{27}$ have been included in the inset to Figure 6B. Additionally, we acknowledge that for the results in Figure $6 \mathrm{~A}$ and $\mathrm{B}$, the range for $\mathrm{Wi}$ in simulations (from 2400 to 47000 ) and experiments (from 30 to 600 ) does not overlap. However, the right trend for the TC or stress values as a function of strain and strain rate is observed ${ }^{27}$ (i.e., for a given $\lambda$, lower stress is observed with decreasing deformation rate or Wi number).

Figure $6 \mathrm{~A}$ and $\mathrm{B}$ make it clear that it is difficult to compare simulations and experiments in terms of strain (or stretch ratio) and strain rate. However, the range of TC and stress values in our simulation results allows us to compare the validation of the STR with both simulation and experimental results. In Figure 7, we test the STR by combining the TC and stress-strain results presented in Figure $6 \mathrm{~A}$ and $\mathrm{B}$, respectively. A linear fit to the simulation results gives a value of $C_{t} G_{N}=$ $0.01 \pm 0.005$. This result is on the same order of magnitude as the experimental value $C_{\mathrm{t}} G_{\mathrm{N}}(\exp )=0.033 \pm 0.002,{ }^{27}$ and, given the numerical uncertainty, it represents a promising validation of the STR via MD simulations for this specific chemical constitution. Note that, as in Figure 6A, we have employed a secondary $y$-axis (but the same scale) in Figure 7 to include the TD experimental results. To compare simulation and experimental results in Figures 6A and 7, we have assumed both the specific heat and the density of the melts to be independent of deformation. We have shown in the insets to Figures $1 \mathrm{~B}$ and $3 \mathrm{~B}$ that the variations in density are not significant in the range of deformations studied in our simulations. Taking the specific heat to be deformation independent is equivalent to adopting the hypothesis of purely entropic elasticity. ${ }^{85}$ Although this hypothesis has been recently contested in a lightly cross-linked natural rubber system, ${ }^{86}$ we consider that the small changes in heat capacity 


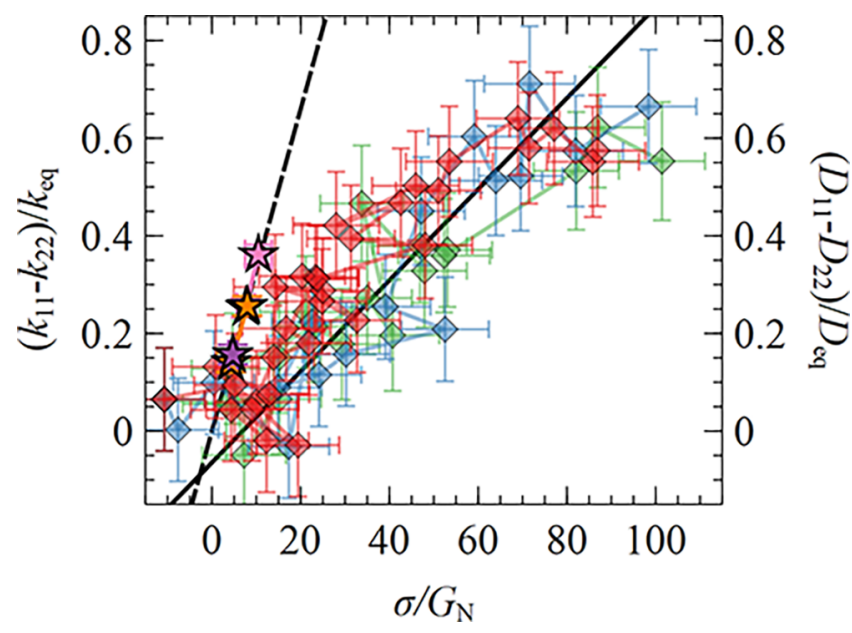

Figure 7. Test of the STR for PS10 $\times 300$ using the results for TC and relaxed stress in Figure 6. The simulation results from this study are represented by diamonds with $\mathrm{Wi}=2400$ (red), $\mathrm{Wi}=4700$ (blue), and $\mathrm{Wi}=47000$ (green). Experimental results by Gupta et al. $^{27}$ are represented by stars with purple $(\mathrm{Wi}=30)$, orange $(\mathrm{Wi}=$ $150)$, and pink $(\mathrm{Wi}=600)$ filling. The solid line represents a linear fit to the simulation data with $C_{t} G_{N}=0.01 \pm 0.005$, where the entanglement plateau modulus $G_{\mathrm{N}}=0.2 \mathrm{MPa}{ }^{75}$ The dashed line is given by the experimental value $\left(C_{t} G_{N}=0.033 \pm 0.002\right) .{ }^{27}$

due to energetic contributions to elasticity are negligible in comparison to the large numerical uncertainty of the simulated results. Finally, the proportionality between anisotropy in TC and stress seems to hold over the whole range of deformations studied for PS (i.e., $\sigma / G_{\mathrm{N}}$ up to 100 ). In contrast, failure of the stress-optic rule in polystyrene has been reported for $\sigma / G_{N} \geq$ 7.5. ${ }^{87}$ We observed similar results for the thermal conductivity and anisotropy of single parent chain PS melts (see Figure S5 in the Supporting Information), indicating that the considered stress-strain and thermal transport properties are not affected at the segmental level.

To our knowledge, all previous MD investigations of anisotropy in thermal conductivity induced by deformation in polymer melts did not characterize the stress in the samples as a function of deformation and therefore were unable to test compliance with the STR. We acknowledge that the large fluctuations in the stress during simulations make estimation of the system stress somewhat problematic and only possible due to the separation of time scales for the different relaxation processes triggered by deformation. Still, the superposition of the anisotropy in TC as a function of stress for different deformation rates for the two studied chemical constitutions further supports our methodology.

Finally, we evaluate the key hypothesis of preferential energy transport along the chain backbone by studying the TC dependence on the molecular weight (or average chain length $\bar{N})$ of well-equilibrated polyethylene samples. This hypothesis was formulated based on early experimental evidence by Hansen et al. ${ }^{16}$ of a proportionality between TC and $N^{1 / 2}$ up to chain lengths much higher than the critical molecular weight $N_{\mathrm{c}}=2 N_{\mathrm{e}}$, where $N_{\mathrm{e}} \sim 80$ is given by the entanglement molecular weight $\left(M_{\mathrm{e}}=1650 \mathrm{~g} / \mathrm{mol} \text { for polyethylene }\right)^{73,75,88}$ Later molecular dynamics simulation studies by Zhao et al., ${ }^{17}$ Zhang et al., ${ }^{18}$ and Wei et al. ${ }^{19}$ indicated that the thermal conductivity reaches a plateau at lower chain lengths $\left(\sim N_{c}\right)$, contradicting the study by Hansen et al. ${ }^{16}$ However, there was no overlap between the range of $N$ studied in experiments ${ }^{16}$ and simulations. ${ }^{17-19}$ The missing gap in chain lengths between experiments and simulations was dictated by two reasons: (1) commercially available low polydispersity melts with low molecular weight are costly, and (2) the computational effort required to equilibrate melts using $\mathrm{MD}$ increases very steeply with the molecular weight of chains. In our study, we overcome the computational cost by equilibrating our samples using CAMC, ${ }^{49}$ making equilibration of samples much faster (more details are given in the Simulation Methods section and the Supporting Information). In Figure 8, we

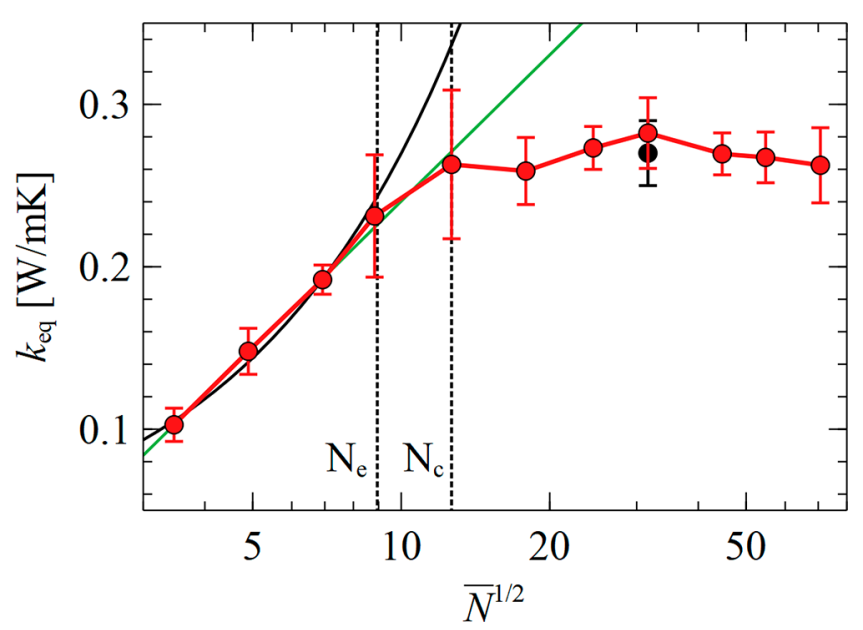

Figure 8. Equilibrium (undeformed) thermal conductivity of $\mathrm{PE}$ melts as a function of average chain length. The solid lines correspond to a fit to the data below $N_{\mathrm{e}}$ to $k_{\text {eq }} \propto \bar{N}^{1 / 2}$ (black) and $k_{\text {eq }} \propto \log \overline{\mathrm{N}}$ (green). The two dashed lines represent the entanglement $N_{\mathrm{e}}$ and critical $N_{c}$ chain lengths. The single black circle corresponds to the equilibrium value for the PE $8 \times 1000$ monodisperse system used in the stretching simulations.

present the equilibrium TC of polyethylene systems with average chain length varying from $\bar{N}=12$ to 5000. An additional speedup in the equilibration of samples is obtained by allowing a small degree of polydispersity (PDI $\leq 1.08$ ), which has a negligible effect on the TC of the system. The single black dot in Figure 8 corresponds to the TC of the monodisperse system PE $8 \times 1000$. Due to differences in the employed force field, the simulation studies mentioned above have determined different absolute values for the equilibrium TC. For example, for systems with average chain lengths above $N_{\mathcal{c}}$, we observe an average TC plateau value $\bar{k}_{\text {eq }}\left(N>N_{\mathrm{c}}\right)=0.27$ $\pm 0.02 \mathrm{~W} / \mathrm{m} \mathrm{K}$, while Zhao et al. ${ }^{17}$ obtain $\bar{k}_{\mathrm{eq}}\left(N>N_{\mathrm{c}}\right) \sim 0.10$ $\mathrm{W} / \mathrm{m} \mathrm{K}$, Zhang et al. ${ }^{18}$ reach $\bar{k}_{\text {eq }}\left(N>N_{\mathrm{c}}\right) \sim 0.17 \mathrm{~W} / \mathrm{m} \mathrm{K}$, and Wei et al. ${ }^{19}$ show $\bar{k}_{\text {eq }}\left(N>N_{\mathrm{c}}\right) \sim 0.24 \mathrm{~W} / \mathrm{m} \mathrm{K}$. It is noteworthy that our simulation results, as well as those in the simulation studies mentioned above, ${ }^{17-19}$ show that the plateau in TC occurs around the critical chain length $N_{\mathcal{c}}$, which is commonly used as the chain length around which the effect of entanglements begins to govern the macroscopic rheological properties of linear polymers. ${ }^{58}$ In our simulation results, we observe that $N^{1 / 2}$ scaling proposed by Hansen et al. ${ }^{16}$ describes the TC data up to $N_{\mathrm{e}}$ reasonably well; between $N_{\mathrm{e}}$ and $N_{\mathcal{O}}$ there is a transition region, and thereafter the TC remains constant. We also find that both the short chains $\left(N<N_{\mathrm{e}}\right)$ and the transition $\left(N_{\mathrm{e}}<N<N_{\mathrm{c}}\right)$ regions are better described by logarithmic scaling $\left(k_{\text {eq }} \propto \log N\right)$. The plateau in TC for $N>$ $N_{\mathrm{c}}$ indicates that the spacing between entanglements becomes the relevant length scale for phonon dispersion (i.e., energy 
that is being transported along a given chain scatters upon reaching an entanglement). The specific mechanism in which entanglements affect the path followed by phonons in the transport of energy remains unclear and will be the subject of future studies. A recent study by Duan et al.$^{89}$ has shown that introducing "kinks" in single polyethylene chains increases the scattering of phonons and thus reduces the TC of a single chain. Furthermore, the degree of chain rotation, which is reduced by topological constraints such as entanglements, has been shown to significantly affect the TC of chains ${ }^{90}$ (i.e., hindered chain rotation results in lower thermal conductivity). Our simulation results provide a region of overlap with the experimental results by Hansen et al. ${ }^{16}(N \geq 600)$ that remained unexplored in previous simulation studies of TC dependence on $N$. We observe a clear discrepancy between the trend in the original experimental results ${ }^{16}$ (TC keeps increasing) and our simulations (TC remains constant) after $N_{c}$. We suspect that the increasing trend in thermal conductivity with chain length in the samples studied experimentally beyond $N_{c}$ could be the result of experimental artifacts. For example, only the four highest molecular weights studied experimentally correspond to "pure" PE; the other molecular weights are obtained by dilution with a PE oligomer of $350 \mathrm{~g} / \mathrm{mol} .{ }^{16}$ Furthermore, it is notable that TC for the pure $\mathrm{PE}$ samples is within the uncertainty of the measurements ${ }^{16}$ (i.e., no clear trend in the TC of the pure PE samples can be observed), and only the samples prepared by dilution in lowmolecular-weight PE show increasing TC with $N$ beyond $N_{\mathrm{c}}$.

\section{CONCLUSIONS}

We have investigated the stress-thermal rule, a linear relationship between thermal conductivity and stress tensors, via molecular dynamics simulations using a united-atom level of description. We propose a scheme to determine the stress of simulated PE and PS systems that have been deformed via nonequilibrium molecular dynamics. Taking advantage of the separation of time scales in the relaxation processes triggered by deformation of the melts $\left(\tau_{0}, \tau_{\mathrm{e}}, \tau_{\mathrm{R}}\right.$, and $\left.\tau_{\mathrm{m}}\right)$, we are able to allow relaxation of local contributions to the stress due to the rapid stretching without significant relaxation of the entangled network stress taking place. The proposed method for simultaneous determination of the TC and stress tensors of entangled polymeric melts subjected to deformation has allowed us to present the first MD study supporting the stress-thermal rule (i.e., $\mathbf{k} \propto \boldsymbol{\tau}$ ) and its extended range of applications $\left(\sigma / G_{\mathrm{N}} \leq 10\right.$ for PE and $\sigma / G_{\mathrm{N}} \leq 100$ for PS). Our finding of stress-thermal coefficients $C_{t} G_{N}$ that are independent of the strain rate (in agreement with experimental evidence ${ }^{27}$ ) further supports our approach to determining the stress. On the other hand, we find that polyethylene, a system for which the STR has not been tested experimentally, shows a normalized stress-thermal coefficient $C_{t} G_{N} 10$ times higher than those observed in other melts and cross-linked elastomers. This finding is in direct contradiction with the proposed universality of the STR, which is based on the available experimental data found for a number of polymers of different chemical constitutions studied by Venerus and co-workers. $^{27,28,35-38}$ We hypothesize that the high degree of entanglement interactions $\left(G_{\mathrm{N}}=2.1 \mathrm{MPa}\right)$ in linear polyethylene due to its very simple macromolecular structure is the likely source for the enhanced correlation between TC and stress tensors. To test this notion, we have performed a study of an entangled polystyrene melt, a material displaying a much lower entanglement plateau $\left(G_{\mathrm{N}}=0.2 \mathrm{MPa}\right)$ and for which experimental confirmation of the STR is available. We apply the same approach to test the STR and find qualitative agreement for all of the results found for PE but a normalized stress-thermal coefficient on the same order of magnitude as in experiments. ${ }^{27}$ It is also noteworthy that in our simulations we obtain good estimates for the equilibrium (unstretched) TC for both polyethylene and polystyrene melts.

Finally, we have tested the key hypothesis used to introduce the stress-thermal rule: preferential transport along the backbone of the chain. This hypothesis was formulated upon the experimental observation of a molecular weight dependence of the TC. We observe an increasing trend $k_{\text {eq }} \propto N^{1 / 2}$ (or $\left.k_{\text {eq }} \propto \log N\right)$ up to the entanglement molecular chain length $N_{e}$, supporting the said hypothesis. However, beyond the critical chain length $N_{\mathrm{c}}=2 N_{\mathrm{e}}$, the thermal conductivity remains constant, indicating that the spacing between entanglement interactions along the chain becomes the limiting length scale for the transport of energy (i.e., making subsequent increases in the chain length becomes irrelevant from a thermal energy transport perspective). This evidence further supports our explanation for the dependence of the normalized stress-thermal coefficient on the density or spacing of entanglements along a chain in the melt, which also determines the entanglement plateau modulus $G_{\mathrm{N}}$.

Our work shows how MD simulations can be used as an effective path to generate trustworthy TC and stress-thermal coefficients that can then be used to model the temperature field in macroscopic flow simulations of polymer melts to help guide manufacturing processes. We acknowledge that to gain a better understanding of the mechanisms behind thermal transport in amorphous polymers, it will be desirable to extend this work to different deformation modes and to lower deformation rates. A follow-up publication, looking at sheared polyethylene samples as well as samples subjected to a deforming field during the MC equilibration method, which allows us to access the steady-state configurations at lower $\mathrm{Wi}$ numbers, is underway.

\section{ASSOCIATED CONTENT}

\section{Supporting Information}

The Supporting Information is available free of charge at https://pubs.acs.org/doi/10.1021/acs.macromol.9b02088.

Details regarding the $\mathrm{G}-\mathrm{K}$ method, behavior of TC around the glass-transition temperature and the optical anisotropy in the studied samples; important checks on equilibration of the melts and system size and manybody interaction effects (PDF)

\section{AUTHOR INFORMATION}

\section{Corresponding Author}

David Nieto Simavilla - Universidad de Burgos, Burgos 09006, Spain; Basque Center for Applied Mathematics, Bilbao 48009, Spain; ㅇo orcid.org/0000-0001-5389-4827; Email: dnieto@ bcamath.org

\section{Authors}

Aristotelis P. Sgouros - National Technical University of Athens, Zografou 15780, Greece; (1) orcid.org/0000-00034183-686X

Georgios G. Vogiatzis - Eindhoven University of Technology, Eindhoven 5612 AZ, Netherlands 
Christos Tzoumanekas - National Technical University of Athens, Zografou 15780, Greece

Vasilis Georgilas - National Technical University of Athens, Zografou 15780, Greece; ○ orcid.org/0000-0003-2999-0514

Wilco M. H. Verbeeten - Universidad de Burgos, Burgos 09006, Spain

Doros N. Theodorou - National Technical University of Athens, Zografou 15780, Greece; (1) orcid.org/0000-00024763-9739

Complete contact information is available at:

https://pubs.acs.org/10.1021/acs.macromol.9b02088

\section{Notes}

The authors declare no competing financial interest.

\section{ACKNOWLEDGMENTS}

The work presented in this study was funded from the European Union's Horizon 2020 research and innovation program under the Marie Skłodowska-Curie IF MTCIATTP 750985 .

\section{REFERENCES}

(1) Favero, J. L.; Secchi, A. R.; Cardozo, N. S. M.; Jasak, H. Viscoelastic Flow Analysis Using the Software OpenFOAM and Differential Constitutive Equations. J. Non-Newtonian Fluid Mech. 2010, 165, 1625-1636.

(2) Verbeeten, W. M. H.; Peters, G. W. M.; Baaijens, F. P. T. Differential Constitutive Equations for Polymer Melts: The Extended Pom-Pom Model. J. Rheol. 2001, 45, 823-843.

(3) Likhtman, A. E.; Graham, R. S. Simple Constitutive Equation for Linear Polymer Melts Derived from Molecular Theory: Rolie-Poly Equation. J. Non-Newtonian Fluid Mech. 2003, 114, 1-12.

(4) Narimissa, E.; Wagner, M. H. Review on Tube Model Based Constitutive Equations for Polydisperse Linear and Long-Chain Branched Polymer Melts. J. Rheol. 2019, 63, 361-375.

(5) Verbeeten, W. M. H.; Peters, G. W. M.; Baaijens, F. P. T. Numerical Simulations of the Planar Contraction Flow for a Polyethylene Melt Using the XPP Model. J. Non-Newtonian Fluid Mech. 2004, 117, 73-84.

(6) Peters, G. W. M.; Baaijens, F. P. T. Modelling of Non-Isothermal Viscoelastic Flows. J. Non-Newtonian Fluid Mech. 1997, 68, 205-224.

(7) Wapperom, P.; Hulsen, M. A. Thermodynamics of Viscoelastic Fluids: The Temperature Equation. J. Rheol. 1998, 42, 999-1019.

(8) Inkson, N. J.; Phillips, T. N.; van Os, R. G. M. Numerical Simulation of Flow Past a Cylinder Using Models of XPP Type. J. Non-Newtonian Fluid Mech. 2009, 156, 7-20.

(9) Oishi, C. M.; Martins, F. P.; Tomé, M. F.; Cuminato, J. A.; McKee, S. Numerical Solution of the EXtended Pom-Pom Model for Viscoelastic Free Surface Flows. J. Non-Newtonian Fluid Mech. 2011, $166,165-179$.

(10) Nieto Simavilla, D.; Verbeeten, W. M. H. In A Combination of the EXtended Pom-Pom Model and the Stress-Thermal Rule to Predict Anisotropy in Thermal Conductivity in Non-Linear Polymeric Flows, AIP Conference Proceedings; AIP Publishing LLC, 2019; Vol. 2139, pp 040004.

(11) Choy, C. L.; Luk, W. H.; Chen, F. C. Thermal Conductivity of Highly Oriented Polyethylene. Polymer 1978, 19, 155-162.

(12) Choy, C. L.; Wong, Y. W.; Yang, G. W.; Kanamoto, T. Elastic Modulus and Thermal Conductivity of Ultradrawn Polyethylene. J. Polym. Sci., Part B: Polym. Phys. 1999, 37, 3359-3367.

(13) Mergenthaler, D. B.; Pietralla, M.; Roy, S.; Kilian, H. G. Thermal Conductivity in Ultraoriented Polyethylene. Macromolecules 1992, 25, 3500-3502.

(14) van den Brule, B. H. A. A. A Network Theory for the Thermal Conductivity of an Amorphous Polymeric Material. Rheol. Acta 1989, $28,257-266$.
(15) Crist, B.; Hereña, P. G. Molecular Orbital Studies of Polyethylene Deformation. J. P. Polym. Sci., Part B: Polym. Phys. 1996, 34, 449-457.

(16) Hansen, D.; Ho, C. C. Thermal Conductivity of High Polymers. J. Polym. Sci., Part A: Gen. Pap. 1965, 3, 659-670.

(17) Zhao, J.; Jiang, J.-W.; Wei, N.; Zhang, Y.; Rabczuk, T. Thermal Conductivity Dependence on Chain Length in Amorphous Polymers. J. Appl. Phys. 2013, 113, No. 184304.

(18) Zhang, T.; Luo, T. Role of Chain Morphology and Stiffness in Thermal Conductivity of Amorphous Polymers. J. Phys. Chem. B 2016, 120, 803-812.

(19) Wei, X.; Luo, T. Chain Length Effect on Thermal Transport in Amorphous Polymers and A Structure-Thermal Conductivity Relation. Phys. Chem. Chem. Phys. 2019, 21, 15523-15530.

(20) He, J.; Kim, K.; Wang, Y.; Liu, J. Strain Effects on the Anisotropic Thermal Transport in Crystalline Polyethylene. Appl. Phys. Lett. 2018, 112, No. 051907.

(21) Li, X.; Maute, K.; Dunn, M. L.; Yang, R. Strain Effects on the Thermal Conductivity of Nanostructures. Phys. Rev. B 2010, 81, No. 245318.

(22) Robbins, A. B.; Minnich, A. J. Crystalline Polymers with Exceptionally Low Thermal Conductivity Studied Using Molecular Dynamics. Appl. Phys. Lett. 2015, 107, No. 201908.

(23) Lu, T.; Kim, K.; Li, X.; Zhou, J.; Chen, G.; Liu, J. Thermal Transport in Semicrystalline Polyethylene by Molecular Dynamics Simulation. J. Appl. Phys. 2018, 123, No. 015107.

(24) Algaer, E. A.; Alaghemandi, M.; Böhm, M. C.; Müller-Plathe, F. Anisotropy of the Thermal Conductivity of Stretched Amorphous Polystyrene in Supercritical Carbon Dioxide Studied by Reverse Nonequilibrium Molecular Dynamics Simulations. J. Phys. Chem. B 2009, 113, 14596-14603.

(25) Terao, T.; Lussetti, E.; Müller-Plathe, F. Nonequilibrium Molecular Dynamics Methods for Computing the Thermal Conductivity: Application to Amorphous Polymers. Phys. Rev. E 2007, 75, No. 057701.

(26) Liu, J.; Yang, R. Tuning the Thermal Conductivity of Polymers with Mechanical Strains. Phys. Rev. B 2010, 81, No. 174122.

(27) Gupta, S.; Schieber, J. D.; Venerus, D. C. Anisotropic Thermal Conduction in Polymer Melts in Uniaxial Elongation Flows. J. Rheol. 2013, 57, 427-439.

(28) Schieber, J. D.; Venerus, D. C.; Gupta, S. Molecular Origins of Anisotropy in the Thermal Conductivity of Deformed Polymer Melts: Stress versus Orientation Contributions. Soft Matter 2012, 8, 11781.

(29) Cahill, D. G.; Watson, S. K.; Pohl, R. O. Lower Limit to the Thermal Conductivity of Disordered Crystals. Phys. Rev. B 1992, 46, $6131-6140$

(30) Xie, X.; Li, D.; Tsai, T.-H.; Liu, J.; Braun, P. V.; Cahill, D. G. Thermal Conductivity, Heat Capacity, and Elastic Constants of Water-Soluble Polymers and Polymer Blends. Macromolecules 2016, 49, 972-978.

(31) Bruns, D.; de Oliveira, T. E.; Rottler, J.; Mukherji, D. Tuning Morphology and Thermal Transport of Asymmetric Smart Polymer Blends by Macromolecular Engineering. Macromolecules 2019, 52, 5510-5517.

(32) Venerus, D. C.; Nieto Simavilla, D.; Schieber, J. D. Thermal transport in cross-linked elastomers subjected to elongational deformations. Rubber Chem. Technol. 2019, 92, 639-652.

(33) Venerus, D. C.; Agarwal, A. Thermal Conductivity of Poly(LLactic Acid) Subjected to Elongational Deformations. J. Polym. Sci., Part B: Polym. Phys. 2019, 57, 547-553.

(34) Sliozberg, Y. R.; Yeh, I.-C.; Kröger, M.; Masser, K. A.; Lenhart, J. L.; Andzelm, J. W. Ordering and Crystallization of Entangled Polyethylene Melts under Uniaxial Tension: A Molecular Dynamics Study. Macromolecules 2018, 51, 9635-9648.

(35) Venerus, D. C.; Kolev, D. N. Anisotropic Thermal Conductivity in Cross-Linked Polybutadienes Subjected to Uniaxial Elongation. Macromolecules 2009, 42, 2594-2598.

(36) Simavilla, D. N.; Schieber, J. D.; Venerus, D. C. Anisotropic Thermal Transport in a Crosslinked Polyisoprene Rubber Subjected 
to Uniaxial Elongation. J. Polym. Sci., Part B: Polym. Phys. 2012, 50, $1638-1644$.

(37) Simavilla, D. N.; Venerus, D. C. Investigation of Anisotropic Thermal Conductivity in Polymers Using Infrared Thermography. J. Heat Transfer 2014, 136, No. 111303.

(38) Venerus, D. C.; Schieber, J. D.; Iddir, H.; Guzmán, J.; Broerman, A. Anisotropic Thermal Diffusivity Measurements in Deforming Polymers and the Stress-Thermal Rule. Int. J. Thermophys. 2001, 22, 1215-1225.

(39) Seyf, H. R.; Yates, L.; Bougher, T. L.; Graham, S.; Cola, B. A.; Detchprohm, T.; Ji, M.-H.; Kim, J.; Dupuis, R.; Lv, W.; et al. Rethinking Phonons: The Issue of Disorder. npj Comput. Mater. 2017, 3, No. 49.

(40) Lv, W.; Henry, A. Phonon Transport in Amorphous Carbon Using Green - Kubo Modal Analysis. Appl. Phys. Lett. 2016, 108, No. 181905.

(41) Ramos, J.; Vega, J. F.; Martínez-Salazar, J. Predicting Experimental Results for Polyethylene by Computer Simulation. Eur. Polym. J. 2018, 99, 298-331.

(42) Vogiatzis, G. G.; Theodorou, D. N. Local Segmental Dynamics and Stresses in Polystyrene-C ${ }_{60}$ Mixtures. Macromolecules 2014, 47, 387-404.

(43) Plimpton, S. Fast Parallel Algorithms for Short-Range Molecular Dynamics. J. Comput. Phys. 1995, 117, 1-19.

(44) Tzoumanekas, C.; Theodorou, D. N. Topological Analysis of Linear Polymer Melts: A Statistical Approach. Macromol. Chem. Phys. 2006, 39, 4592-4604.

(45) Fetters, L. J.; Lohse, D. J.; Colby, R. H. Chain Dimensions and Entanglement Spacings. Physical Properties of Polymers Handbook; Springer New York: New York, NY, 2007; pp 447-454.

(46) Martin, M. G.; Siepmann, J. I. Transferable Potentials for Phase Equilibria. 1. United-Atom Description of n-Alkanes. J. Phys. Chem. B 1998, 102, 2569-2577.

(47) Martin, M. G.; Siepmann, J. I. Novel Configurational-Bias Monte Carlo Method for Branched Molecules. Transferable Potentials for Phase Equilibria. 2. United-Atom Description of Branched Alkanes. J. Phys. Chem. B 1999, 103, 4508-4517.

(48) Maerzke, K. A.; Siepmann, J. I. Transferable Potentials for Phase Equilibria-Coarse-Grain Description for Linear Alkanes. J. Phys. Chem. B 2011, 115, 3452-3465.

(49) Mavrantzas, V. G.; Theodorou, D. N. Atomistic Simulation of Polymer Melt Elasticity: Calculation of the Free Energy of an Oriented Polymer Melt. Macromolecules 1998, 31, 6310-6332.

(50) Karayiannis, N. C.; Mavrantzas, V. G.; Theodorou, D. N. A Novel Monte Carlo Scheme for the Rapid Equilibration of Atomistic Model Polymer Systems of Precisely Defined Molecular Architecture. Phys. Rev. Lett. 2002, 88, No. 105503.

(51) Ramos, J.; Peristeras, L. D.; Theodorou, D. N. Monte Carlo Simulation of Short Chain Branched Polyolefins in the Molten State. Macromolecules 2007, 40, 9640-9650.

(52) Karayiannis, N. C.; Kröger, M. Combined Molecular Algorithms for the Generation, Equilibration and Topological Analysis of Entangled Polymers: Methodology and Performance. Int. J. Mol. Sci. 2009, 10, 5054-5089.

(53) Theodorou, D. N. Understanding and Predicting StructureProperty Relations in Polymeric Materials through Molecular Simulations. Mol. Phys. 2004, 102, 147-166.

(54) Sgouros, A. P.; Vogiatzis, G. G.; Kritikos, G.; Boziki, A.; Nikolakopoulou, A.; Liveris, D.; Theodorou, D. N. Molecular Simulations of Free and Graphite Capped Polyethylene Films: Estimation of the Interfacial Free Energies. Macromolecules 2017, 50, 8827-8844.

(55) Sgouros, A. P.; Megariotis, G.; Theodorou, D. N. Slip-Spring Model for the Linear and Nonlinear Viscoelastic Properties of Molten Polyethylene Derived from Atomistic Simulations. Macromolecules 2017, 50, 4524-4541.

(56) Zhang, G.; Chazirakis, A.; Harmandaris, V. A.; Stuehn, T.; Daoulas, K. C.; Kremer, K. Hierarchical Modelling of Polystyrene
Melts: From Soft Blobs to Atomistic Resolution. Soft Matter 2019, 15, 289-302.

(57) Spyriouni, T.; Tzoumanekas, C.; Theodorou, D. N.; MüllerPlathe, F.; Milano, G. Coarse-Grained and Reverse-Mapped UnitedAtom Simulations of Long-Chain Atactic Polystyrene Melts: Structure, Thermodynamic Properties, Chain Conformation, and Entanglements. Macromolecules 2007, 40, 3876-3885.

(58) Doi, M. Masao.; Edwards, S. F. Sam F. The Theory of Polymer Dynamics; Clarendon Press, 1986.

(59) Mathioudakis, I. G.; Vogiatzis, G. G.; Tzoumanekas, C.; Theodorou, D. N. Molecular Modeling and Simulation of Polymer Nanocomposites at Multiple Length Scales. IEEE Trans. Nanotechnol. 2016, 15, 416-422.

(60) Lyulin, A. V.; Michels, M. A. J. Molecular Dynamics Simulation of Bulk Atactic Polystyrene in the Vicinity of Tg. Macromolecules 2002, 35, 1463-1472.

(61) Lempesis, N.; Vogiatzis, G. G.; Boulougouris, G. C.; van Breemen, L. C. A.; Hütter, M.; Theodorou, D. N. Tracking a Glassy Polymer on Its Energy Landscape in the Course of Elastic Deformation. Mol. Phys. 2013, 111, 3430-3441.

(62) Nosé, S. A Unified Formulation of the Constant Temperature Molecular Dynamics Methods. J. Chem. Phys. 1984, 81, 511-519.

(63) Hoover, W. G. Canonical Dynamics: Equilibrium Phase-Space Distributions. Phys. Rev. A 1985, 31, 1695-1697.

(64) Martyna, G. J.; Tobias, D. J.; Klein, M. L. Constant Pressure Molecular Dynamics Algorithms. J. Chem. Phys. 1994, 101, 41774189.

(65) Savitzky, A.; Golay, M. J. E. Smoothing and Differentiation of Data by Simplified Least Squares Procedures. Anal. Chem. 1964, 36, 1627-1639.

(66) Dealy, J. M.; Larson, R. G. Structure and Rheology of Molten Polymers: From Structure to Flow Behavior and Back Again; Hanser Publishers, 2006.

(67) Harmandaris, V. A.; Mavrantzas, V. G. Segmental Dynamics in Polyethylene Melts through Atomistic Molecular Dynamics Simulations. Recent Research Topics and Developments in Chemical Physics: From Quantum Scale to Macroscale; Transworld Research Network: 2008; 661, 179-196. ISBN: 978-81-7895-316-8.

(68) Wagner, M. H.; Kheirandish, S.; Hassager, O. Quantitative Prediction of Transient and Steady-State Elongational Viscosity of Nearly Monodisperse Polystyrene Melts. J. Rheol. 2005, 49, 13171327.

(69) Stratton, R. A. The Dependence of Non-Newtonian Viscosity on Molecular Weight for "Monodisperse" Polystyrene. J. Colloid Interface Sci. 1966, 22, 517-530.

(70) Wang, J.; Porter, R. S. On the Viscosity-Temperature Behavior of Polymer Melts. Rheol. Acta 1995, 34, 496-503.

(71) Hsu, H.-P.; Kremer, K. Static and Dynamic Properties of Large Polymer Melts in Equilibrium. J. Chem. Phys. 2016, 144, No. 154907.

(72) Ramos, J.; Vega, J. F.; Martínez-Salazar, J. Molecular Dynamics Simulations for the Description of Experimental Molecular Conformation, Melt Dynamics, and Phase Transitions in Polyethylene. Macromolecules 2015, 48, 5016-5027.

(73) Vega, J. F.; Rastogi, S.; Peters, G. W. M.; Meijer, H. E. H. Rheology and Reptation of Linear Polymers. Ultrahigh Molecular Weight Chain Dynamics in the Melt. J. Rheol. 2004, 48, 663-678.

(74) Nafar Sefiddashti, M. H.; Edwards, B. J.; Khomami, B. Individual Chain Dynamics of a Polyethylene Melt Undergoing Steady Shear Flow. J. Rheol. 2015, 59, 119-153.

(75) Fetters, L. J.; Lohse, D. J.; Richter, D.; Witten, T. A.; Zirkel, A. Connection between Polymer Molecular Weight, Density, Chain Dimensions, and Melt Viscoelastic Properties. Macromolecules 1994, 27, 4639-4647.

(76) Hsu, H.-P.; Kremer, K. Primitive Path Analysis and Stress Distribution in Highly Strained Macromolecules. ACS Macro Lett. 2018, 7, 107-111.

(77) Evans, D. J.; Morriss, G. P. Statistical Mechanics of Nonequilibrium Liquids; Academic Press, 1990. 
(78) Pegolo, P. Teoria e Simulazione Numerica Della Conducibilità Termica Dell' Acqua; Università degli Studi di Trieste, 2018.

(79) Sheldon, R. P.; Lane, S. K. Thermal Conductivities of Polymers II-Polyethylene. Polymer 1965, 6, 205-212.

(80) Mavrantzas, V. G.; Theodorou, D. N. Atomistic Simulation of the Birefringence of Uniaxially Stretched Polyethylene Melts. Comput. Theor. Polym. Sci. 2000, 10, 1-13.

(81) Tautz, H. Bestimmung Der Warmeleifahigkeit von Kautschukvulkanisaten in Abhangigkeit von Der Dehnung. Exp. Tech. Phys. 1959, 7, 628.

(82) Engelmann, S.; Meyer, J.; Hentschke, R. Computer Simulation of Thermal Conductivity in Vulcanized Polyisoprene at Variable Strain and Temperature. Phys. Rev. B 2017, 96, No. 054110.

(83) Qin, J.; Milner, S. T.; Stephanou, P. S.; Mavrantzas, V. G. Effects of Tube Persistence Length on Dynamics of Mildly Entangled Polymers. J. Rheol. 2012, 56, 707-723.

(84) Physical Properties of Polymers Handbook; Mark, J. E., Ed.; Springer New York: New York, NY, 2007.

(85) Sarti, G. C.; Esposito, N. Testing Thermodynamic Constitutive Equations for Polymers by Adiabatic Deformation Experiments. J. Non-Newtonian Fluid Mech. 1977, 3, 65-76.

(86) Nieto Simavilla, D.; Schieber, J. D.; Venerus, D. C. Evidence of Deformation-Dependent Heat Capacity and Energetic Elasticity in a Cross-Linked Elastomer Subjected to Uniaxial Elongation. Macromolecules 2018, 51, 589-597.

(87) Luap, C.; Müller, C.; Schweizer, T.; Venerus, D. C. Simultaneous Stress and Birefringence Measurements during Uniaxial Elongation of Polystyrene Melts with Narrow Molecular Weight Distribution. Rheol. Acta 2005, 45, 83-91.

(88) Fetters, L. J.; Lohse, D. J.; Milner, S. T.; Graessley, W. W. Packing Length Influence in Linear Polymer Melts on the Entanglement, Critical, and Reptation Molecular Weights. Macromolecules 1999, 32, 6847-6851.

(89) Duan, X.; Li, Z.; Liu, J.; Chen, G.; Li, X. Roles of Kink on the Thermal Transport in Single Polyethylene Chains. J. Appl. Phys. 2019, 125, No. 164303.

(90) Ma, H.; Tian, Z. Chain Rotation Significantly Reduces Thermal Conductivity of Single-Chain Polymers. J. Mater. Res. 2019, 34, 126133. 